\title{
DETERMINANTES DEL PRECIO SPOT ELECTRICO EN EL SISTEMA INTERCONECTADO CENTRAL DE CHILE*
}

\section{THE DETERMINANTS OF ELECTRICITY SPOT PRICE IN THE CENTRAL INTERCONNECTED SYSTEM OF CHILE}

\section{IRYNA SIKORA** \\ KPMG España Asesores S.L.}

\section{JUAN ANTONIO CAMPOS ABAD*** \\ División de Prospectiva y Política Energética, Ministerio de Energía}

\section{JAVIER BUSTOS SALVAGNO****}

División de Prospectiva y Política Energética, Ministerio de Energía

\begin{abstract}
This paper aims to identify the key determinants of Chilean spot power price and assess their relative importance in recent price decline. To do so, we use a VAR model with weekly data between the years 2015-2016 and variance decomposition analysis to attribute variations of marginal cost to different shocks. The results show that while the availability of water for hydropower and lower fuel prices are important for explaining power spot prices, they only account for 50\%-75\% of the marginal cost's variance, leaving 25\%-50\% to other factors, such as more renewable generation and lower generation from oil sources, among other factors.
\end{abstract}

Keywords: VAR, spot price, electricity, Chile.

JEL Classification: C32, L94, Q41, Q48.

\footnotetext{
* El presente documento se ha visto enriquecido por los comentarios recibidos por Mauricio Tejada, Carlos García y Cristóbal Muñoz, así como de los participantes en el 6th Latin American Energy Economics Meeting y el Seminario de la Escuela de Gobierno de la Universidad Adolfo Ibáñez. Este trabajo no representa un documento oficial ni corresponde a una declaración de ningún tipo del Ministerio de Energía ni de KPMG Asesores S.L. Todos los errores son propios de los autores.

** E-mail: isikora@kpmg.es

*** E-mail: jcampos@minenergia.cl

**** E-mail: jbustos@minenergia.cl
} 


\section{Resumen}

Este estudio tiene como objetivo encontrar los determinantes del precio spot de la electricidad en Chile y caracterizar su disminución significativa entre el 2015 y 2016. Para ello se emplea un modelo de VAR con datos semanales 2011-2016 y un análisis de descomposición de varianza de costo marginal ante distintos shocks de las variables del modelo. Los principales resultados muestran que las variables generación hidroeléctrica, y aportes de gas y carbón, explican entre el 50\%-75\% de la varianza del costo marginal, dejando entre $25 \%-50 \%$ para el resto de factores, como incremento de aportes ERNC y menor generación diésel, entre otros.

Palabras clave: VAR, precio spot, electricidad, Chile.

Clasificación JEL: C32, L94, Q41, Q48.

\section{INTRODUCCION}

El costo marginal de la energía eléctrica (o precio spot de electricidad) es uno de los principales indicadores del mercado eléctrico y señal de su condición de adaptación entre oferta y demanda. En términos simples refleja el costo de suministrar un kilo watt hora $(\mathrm{kWh})$ adicional al sistema eléctrico. Además, representa el precio a utilizar en la valorización de las transferencias de energía entre empresas generadoras. En general, el costo marginal se percibe como señal de corto plazo pero a la vez influye en el precio de contratos de suministro de mediano y largo plazo.

Las diferencias entre el precio spot y el precio de los contratos de suministro impactan directamente en la viabilidad financiera de las empresas de generación ${ }^{1}$. El riesgo del precio spot, particularmente después de la volatilidad que se creó producto de la crisis del gas argentino a mediados de la década pasada, llevó a que se extendiera la práctica de la indexación de precios de contratos a costo marginal. La Figura 1 muestra la dinámica del costo marginal en los últimos 10 años.

Varios estudios econométricos confirman la importancia del costo marginal como un punto de referencia en el sector, particularmente a la hora de determinar precios de contratos de suministro de mediano y largo plazo. Roubik y Rudnik (2009), Arellano y Serra (2010), Moreno et al. (2012), Fabra et al. (2014), Varas y Rudnick (2014) y Bustos (2015a) muestran que el costo marginal esperado es uno de los principales determinantes de los precios de contratos en libres y en regulados. Adicionalmente, las divergencias de los costos marginales entre diferentes barras del sistema de transmisión

\footnotetext{
Para ilustrar este punto está el caso de la generadora Campanario con centrales a petróleo diésel que optó por una estrategia de compras en el mercado spot para cumplir con las obligaciones sostenidas en los contratos suscritos con clientes regulados en el 2009. Campanario no pudo sostener su flujo de caja en 2011, ya que el precio spot subió muy por encima de lo esperado por la empresa y respecto del precio establecido en los contratos suscritos.
} 


\section{FIGURA 1}

\section{COSTO MARGINAL SIC 2 2006-16 (USD/MWH), BARRAS QUILLOTA,} CHARRUA Y CARDONES, 220

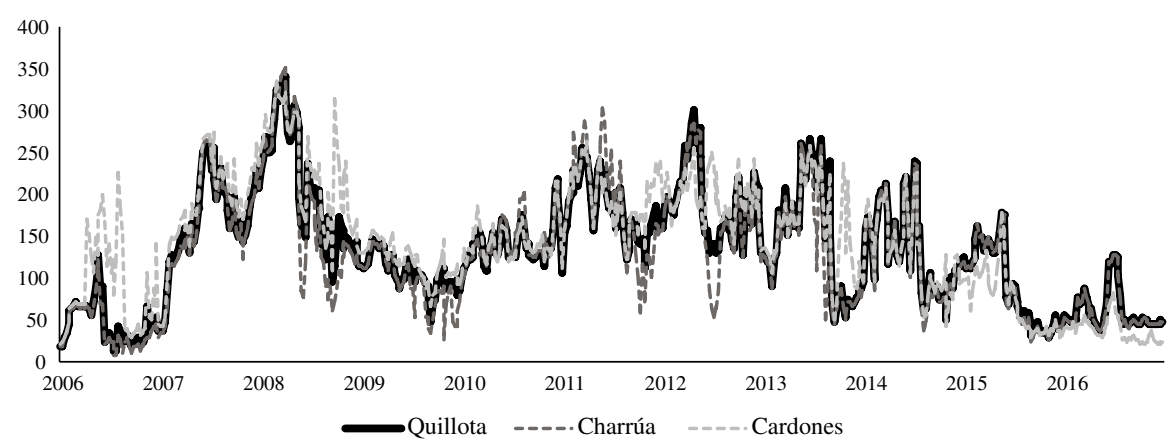

reflejan el estado del mismo, siendo el desacople ${ }^{3}$ de precios un potencial problema financiero para las empresas del sector.

Durante los últimos meses, mucho se ha especulado acerca de los factores que han determinado la importante caída de los costos marginales del Sistema Interconectado Central (SIC). Esa atención es justificada: a modo de ejemplo, el costo marginal eléctrico en la barra Quillota promediaba los 152 USD/MWh en 2013, y bajó a 133 USD/MWh en 2014, a 90 USD/MWh en 2015 y a 60 USD/MWh en 2016 ${ }^{4}$, lo que representa una caída de $60 \%$ en tres años.

Académicos y distintas publicaciones del sector eléctrico ${ }^{5}$ han desarrollado diversas conjeturas con el fin de explicar las verdaderas razones y causalidades a las que se atribuye la caída de los costos marginales durante este período. Penetración de generación renovable no convencional (ERNC), precipitaciones tempranas, caída en el precio de los combustibles fósiles, aumento de disponibilidad de generación térmica a carbón y el crecimiento de la demanda menor que lo esperado, han sido

2 Actualmente en Chile existen dos sistemas eléctricos: Sistema Interconectado Central (SIC) y Sistema Interconectado de Norte Grande (SING), que a partir del 2018 estarán interconectados formando un futuro Sistema Interconectado Nacional. El presente análisis se centra en el Sistema Interconectado Central debido a su mayor tamaño, más diversa matriz de generación y mayor variabilidad en precios spot.

3 Corresponde a la falta de capacidad de transmisión que imposibilita la transferencia de energía, llevando a que la operación del sistema se aleje del óptimo económico del sistema al ser necesario el despacho de unidades de generación de mayor precio.

4 Datos diarios, ponderados por generación horaria del sistema, Centro de Despacho Económico y Carga del Sistema Interconectado Central (CDEC SIC). Se dispone de datos hasta 31 de mayo 2016.

5 Núñez, Ignacio (2015), “¿Por qué ha caído el costo marginal en el Sistema Interconectado Central de Chile?”. Revista Electricidad (2016), “Costos marginales de energía caen $73 \%$ en un año por más ERNC y lluvias tempranas". 
las principales razones que se han imputado a la caída de los costos marginales en el SIC.

La evidencia circunstancial apoya a la mayoría de estos supuestos en los últimos años: efectivamente, la disponibilidad del agua aumentó significativamente ${ }^{6}$, el aporte de la generación renovable no convencional ha crecido más de cuatro veces ${ }^{7}$, los precios de combustibles han caído en más de $40 \%{ }^{8}$, la demanda eléctrica ha crecido 2\%-3\% más lento de lo previsto 9 . Pero en cuanto a la generación a base de carbón, su aporte a la matriz no ha variado significativamente, representando 33\% en 2013 versus $32 \%$ en 2016.

A pesar que las tendencias enunciadas parecen estar alineadas con el resultado esperado de una baja en el costo marginal eléctrico, las hipótesis de la importancia de cada uno de esos factores no han sido testeadas estadísticamente, siendo producto de un análisis descriptivo del sector. Por otro lado, tampoco está asegurada la causalidad de estos factores sobre el costo marginal.

Los objetivos de este trabajo son dos. En primer lugar, proponer una metodología para encontrar los principales determinantes del costo marginal eléctrico en el Sistema Interconectado Central. Hasta el momento, no existe una única metodología establecida para evaluar los principales factores que determinan la evolución de los precios spot eléctricos en Chile, y este trabajo permite una primera aproximación a dicho desafío. En segundo lugar, se pretende testear y conocer la veracidad de los grupos de factores anteriormente mencionados para explicar la significativa disminución de costos marginales en los últimos años. La estimación de la relación entre costo marginal y las características del Sistema Interconectado Central se realiza por medio de un modelo de Vectores Autorregresivos (VAR), considerando datos semanales para el período comprendido entre el 2011 y 2016. Adicionalmente se ha incorporado una estimación mediante un Modelo de Corrección de Error Simple.

Comprender los determinantes de los costos marginales eléctricos es relevante para elaborar diagnósticos consistentes con la realidad, así como diseñar políticas energéticas efectivas tanto en el corto como en el largo plazo. Sin ir más lejos, la primera y una de las principales metas de la actual Agenda de Energía ${ }^{10}$ es reducir el costo marginal eléctrico en $30 \%$. En el SIC esto se traduciría a que el costo marginal

6 La energía promedio embalsada en el conjunto de embalses del SIC era de 1,314 GWh en 2013, 1,775 GWh en 2014 y 2,232 GWh en 2015 -según datos diarios de la energía embalsada, SEC.

7 Generación ERNC ha crecido de 1\% en 2013 a 3\% en 2014, y a 5\% en los años 2015 y 2016 -según datos diarios de generación eléctrica recopilados por la Superintendencia de Electricidad y Combustibles (SEC).

8 Los precios de petróleo diésel, gas natural y carbón en los mercados internacionales han disminuido en más de $40 \%$ entre 2013 y 2015 -según datos Bloomberg diarios de mercados internacionales para petróleo Brent y gas natural Henry Hub, datos de la Comisión Nacional de Energía (CNE) para los precios del carbón.

9 Según datos de Demanda esperada en informes técnicos, fijación de precios de nudo octubre 2013, octubre 2014, CNE versus demanda efectiva en datos diarios de la energía generada en el SIC recopilados por la SEC.

10 Ministerio de Energía (2014), Agenda de Energía, Metas y objetivos de la Agenda de Energía. 
promedio del año 2013 de 151 USD/MWh sea inferior a 106 USD/MWh en el año 2017, en la barra de Quillota.

Este trabajo se estructura en cuatro partes, la primera incluye la revisión de literatura para la realización de este trabajo. La segunda parte continúa con el modelo empírico, en donde se describe el modelo VAR y las variables utilizadas, luego se presenta el análisis de resultados, y por último, el trabajo termina con la discusión y las conclusiones.

\section{LITERATURA}

Al ser tan dinámicos y cambiantes, el enfoque más intuitivo para analizar los mercados energéticos sería mediante el análisis de series de tiempo. Durante la última década se ha desarrollado ampliamente la literatura empírica al respecto aplicando distintos métodos de modelización de regresión de series temporales a los mercados de electricidad y combustibles en todo el mundo. Higgs y Worthington (2010) presentan una revisión extensa de estos métodos de análisis aplicados a los determinantes del precio de combustibles y electricidad, sus dinámicas y su volatilidad, y también sus impactos en distintas dimensiones de la actividad económica del país.

Una rama importante de esta literatura se basa en series de tiempo estacionarias y las técnicas multivariantes. En concreto, los modelos VAR (Vector Autoregression Models) y VEC (Vector Error Correction Models) se están usando para analizar mercados energéticos. Dichos modelos y sus modificaciones se usan permanente y constantemente en el contexto de los mercados de petróleo crudo y de gas natural (por ejemplo Kilian 2009 y 2012; Toews y Naumov, 2015).

La literatura en mercados eléctricos en particular es todavía más escasa que en el caso de combustibles. Sin embargo, los modelos autorregresivos ya se han usado para estimar las relaciones entre los precios de electricidad y los costos de combustibles en Estados Unidos (Mjelde y Bessler, 2009), Europa (Ferkingstad, Loland y Wilhelmsen 2011; Fezzi 2007), y también en América Latina (Correa y García, 2013).

Además, estos modelos se han usado para analizar otros aspectos del mercado eléctrico, como evaluar el impacto de permisos a emisiones y moratoria nuclear (Thoenes 2015), así como para relacionar los precios de electricidad entre los distintos estados de Estados Unidos (Park, Mjelde y Bessler, 2006).

En la literatura chilena aún no existen muchos trabajos al respecto. Morandé y Soto (1996) llevaron a cabo un análisis de los determinantes de los precios de contratos libres en el SIC durante la primer mitad de los 90, no encontrando evidencia que el precio spot o costo marginal afectara el precio de contratos libres. Por el contrario, el precio de contratos regulados y el precio spot estaban cointegrados, por lo que el precio regulado estaría reflejando adecuadamente el costo marginal esperado de largo plazo. Bustos (2015b), por otra parte, encuentra que no es posible rechazar la hipótesis de que el precio spot cause "a la Granger" el precio de contratos libres durante el período 1995-2010. En este caso, los principales determinantes del precio spot encontrados fueron el precio de combustibles y la hidrología del SIC. 


\section{MODELO VAR PARA LA DESCOMPOSICION DEL COSTO MARGINAL}

Para poder atribuir la dinámica del costo marginal a los diferentes factores, utilizamos el modelo de Vectores Autorregresivos VAR. Este modelo, en su esencia, es un sistema de variables, en donde cada variable endógena está representada como una función de su propio pasado y del pasado de otras variables endógenas del sistema. El VAR se caracteriza por incorporar variables que guardan una relación compleja entre ellas, sin necesidad de especificar una relación de equilibrio de largo plazo y permite analizar la interrelación entre las diferentes series de tiempo.

El modelo VAR puede ser pensado como la forma reducida de un sistema económico dinámico que implica un vector de variables. Modelo VAR con $p$ rezagos (VAR $(p))$ se puede escribir como:

$$
\begin{gathered}
A X_{t}=B_{1} X_{t-1}+B_{2} X_{t-2}+\ldots+B_{p} X_{t-p}+u_{t} \\
E_{u u}{ }^{\prime}=\sum=\left[\begin{array}{cccc}
\sigma_{u 1}^{2} & 0 & \ldots & 0 \\
0 & \sigma_{u 2}^{2} & \ldots & 0 \\
& & \ldots & \\
0 & 0 & \ldots & \sigma_{u n}^{2}
\end{array}\right]
\end{gathered}
$$

Debido a que el propósito de este estudio es descomponer la variación del costo marginal entre diferentes factores, el costo marginal es la principal variable de interés, mientras las características del sistema eléctrico tienen un rol de las variables explicativas.

En el Sistema Interconectado Central existen 56 barras (puntos de recepción y distribución de energía eléctrica) de $220 \mathrm{~V}$, que constituyen 56 "mercados locales" de energía eléctrica dispersos geográficamente. Se dispone de los datos de costos marginales para cada una de esas 56 barras, por lo que es posible modelar cada una de ellas. Sin embargo, para el presente análisis de los costos marginales se eligieron seis barras: Diego de Almagro (DA), Cardones (CA), Quillota (QU), Alto Jahuel $(\mathrm{AJ})$, Charrúa $(\mathrm{CH})$ y Puerto Montt $(\mathrm{PM})$. Cada una de esas barras tiene una demanda eléctrica significativa, y además, están geográficamente separadas: Diego de Almagro y Cardones representan el Norte del Sistema Interconectado Central; Quillota y Alto Jahuel representan el Centro; y las barras Charrúa y Puerto Montt representan el Sur del país.

Para estimar el modelo VAR se ha levantado la información sobre las fuerzas determinantes del costo marginal a partir de la estructura del mercado spot de electricidad en Chile. A causa del trade-off entre la cantidad de las variables que componen el modelo VAR, y la precisión de las estimaciones en las muestras relativamente cortas, 
se han elegido cinco características que se perciben más relevantes para ser incluidas al modelo principal y completar el análisis con los modelos auxiliares para explorar los efectos de otras variables del sector.

Denotemos $X_{t}=\left(X_{0 t}, \ldots, X_{4 t}\right)$ el vector de las características del sistema eléctrico chileno en el momento $t$, donde:

$X_{0 t}$ es el costo marginal en la barra de interés en el momento $t$, medido en dólares estadounidenses por megavatio-hora (UDS/MWh).

Las variables $X_{1 t}, X_{2 t}, X_{3 t}$ corresponden a los aportes porcentuales de tecnologías a gas, carbón e hidroelectricidad, respectivamente, para generación eléctrica total en el momento $t$.

La variable $X_{4 t}$ caracteriza la disponibilidad de agua para la generación hidroeléctrica y corresponde al estado de embalses del Sistema Interconectado Central en el momento $t$, medidos en energía embalsada, GWh.

Así, con este vector de cinco variables se estima la ecuación (1). El modelo VAR se estimará en diferencias para todas las variables, ya que la variable estado de embalse no es estacionaria en niveles. Por esta razón, para mantener la estacionariedad de todas las variables del modelo, estas serán estimadas en diferencias y considerando 3 períodos de rezago, lo que significa 21 días o tres semanas ${ }^{11}$.

En virtud del propósito de este análisis, nuestro interés principal está en descomponer la varianza del costo marginal y determinar qué porcentaje de la misma se debe atribuir a cada variable del modelo. Para ello, después de la estimación VAR, pasamos al análisis de la descomposición:

El rezago del costo marginal capturará el efecto de la tendencia o patrón en la propia barra. En general, interpretamos este efecto como el efecto residual, es decir, la parte de variación no explicada por otros factores del modelo.

Los aportes de gas y carbón se usan como aproximaciones para los costos de combustibles para la generación eléctrica (la siguiente sección explica la lógica de esa aproximación). Para efectos de interpretación, esas variables capturan los efectos de variación de precios de gas y carbón en el costo marginal.

El aporte hidroeléctrico, así como estado de embalses reflejan las condiciones meteorológicas o disponibilidad de agua, y su impacto se interpreta como la importancia de condiciones hídricas para las variaciones del costo marginal.

Esta simple composición del modelo nos permite separar los efectos de la disponibilidad de agua para generación eléctrica y los combustibles (los candidatos más populares en explicar la reciente caída del costo marginal) de todo el resto de los factores y tendencias del sector eléctrico.

11 Los detalles de las pruebas de estacionaridad están presentados en el Anexo 1A. Los detalles de las pruebas de rezagos en el Anexo 1B. 
Para profundizar el análisis e introducir los chequeos de sensibilidad, además del modelo inicial, estimamos varios modelos adicionales, considerando las siguientes variables:

- $X_{\mathrm{QU} t}-$ el costo marginal en la barra Quillota en el momento $t$, medido en dólares estadounidenses por megavatio-hora (UDS/MWh). La inclusión de esa variable permite medir el efecto de desacople entre las barras del sistema eléctrico.

- $X_{\mathrm{PASADA}} t$ y $X_{\mathrm{EMBALSE}}$ - aportes porcentuales de hidroeléctrica pasada y embalse, respectivamente, para generación eléctrica total en el momento $t$. La inclusión de esas variables permite diferenciar los impactos de generación hidro entre tecnologías.

- $X_{\mathrm{ERNC} t}$ y $X_{\mathrm{DIESEL} t}$ - aportes porcentuales de ERNC y diésel, respectivamente, para generación eléctrica total en el momento $t$. La inclusión de esas variables permite medir la importancia relativa de mayor generación ERNC y menor despacho de diésel en el costo marginal.

- $X_{\mathrm{D} t}$ - la medida de demanda eléctrica en el momento $t$ : se consideró la demanda absoluta medida en GWh, así como la demanda relativa medida como proporción de la demanda en el momento $t$ sobre la demanda máxima en el período. La inclusión de esa variable permite medir la importancia de menor crecimiento de la demanda en el desarrollo del precio spot.

La inclusión de esas variables permite indagar más profundamente en los factores no explicados por agua ni combustibles, y obtener una mejor idea de cómo se compone el "Resto de factores" representado por el rezago del costo marginal.

\section{Precios de combustibles vs. aportes por tecnología}

Para analizar las variaciones del costo marginal lo más lógico sería incorporar los precios de combustibles a nuestro modelo. Sin embargo, medir correctamente los costos de combustibles reales que enfrentan los generadores no es una tarea fácil. Esto se debe a que no existe información pública al respecto ni son homogéneos entre los generadores, ya que provienen de los contratos privados de cada empresa.

Una posible solución a este problema sería usar los precios de combustibles referenciales, publicados por la Comisión Nacional de Energía (CNE) o disponibles de los mercados internacionales. La Figura 2 muestra gráficamente la relación entre el costo marginal y los precios de referencia de gas y carbón. Según el gráfico, el costo marginal parece estar bastante alineado con los precios de combustibles: en los últimos años se observa la reducción de los tres, costos marginales y ambos precios. Sin embargo, es posible que esta sea una correlación espuria. 


\section{FIGURA 2}

RELACION ENTRE COSTO MARGINAL, PRECIO DE GAS Y PRECIO DE CARBON

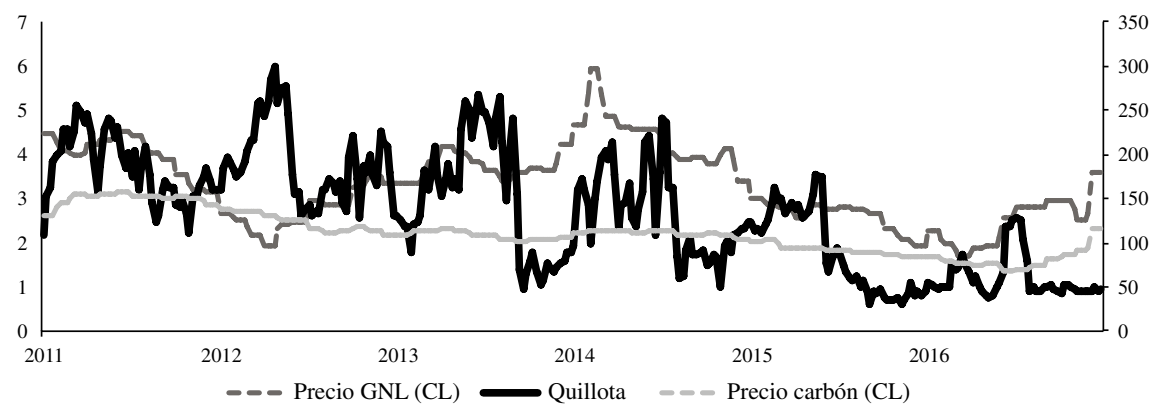

Si los precios de combustibles que observamos se reflejaran en el costo marginal declarado por las generadoras, observaríamos una clara correlación negativa entre los aportes y precios de combustibles: costo de generación más bajo implicaría mayor probabilidad de despacho y, en consecuencia, mayor producción ${ }^{12}$.

La Tabla 1 muestra las correlaciones entre el costo marginal (barra Quillota), aportes y precios de gas y carbón para cada año desde 2011. Observamos que en cuatro años, para el caso de gas y de carbón, la correlación entre precios de combustibles y sus aportes es positiva: en 2014 y 2015 un mayor precio de gas o carbón se traducía en mayores aportes de esas tecnologías.

\section{TABLA 1}

TABLA DE CORRELACIONES ENTRE PRECIOS DE GAS Y CARBON, SUS APORTES Y COSTOS MARGINALES

\begin{tabular}{|c|r|r|r|r|}
\hline \multirow{2}{*}{ Año } & \multicolumn{2}{|c|}{ Gas } & \multicolumn{2}{c|}{ Carbón } \\
\cline { 2 - 5 } & Aporte & Costo marginal & Aporte & Costo marginal \\
\hline 2011 & 0,7645 & 0,3644 & 0,2017 & 0,1754 \\
2012 & $-0,4006$ & $-0,4928$ & $-0,5318$ & 0,3921 \\
2013 & 0,0054 & 0,0775 & 0,0487 & $-0,1258$ \\
2014 & 0,7327 & 0,4386 & 0,4994 & 0,4574 \\
2015 & 0,6589 & 0,6139 & 0,2819 & 0,6893 \\
2016 & $-0,3526$ & $-0,0420$ & $-0,4037$ & $-0,3082$ \\
\hline
\end{tabular}

12 El mercado spot es Chile funciona mediante un despacho centralizado que ordena un Coordinador Eléctrico a partir de los costos declarados por las empresas de generación. De esta manera, unidades de menor costo son despachadas primero siguiendo un orden de menor a mayor costo de operación. 


\section{FIGURA 3}

\section{RELACION ENTRE APORTES DE GAS Y SUS PRECIOS}

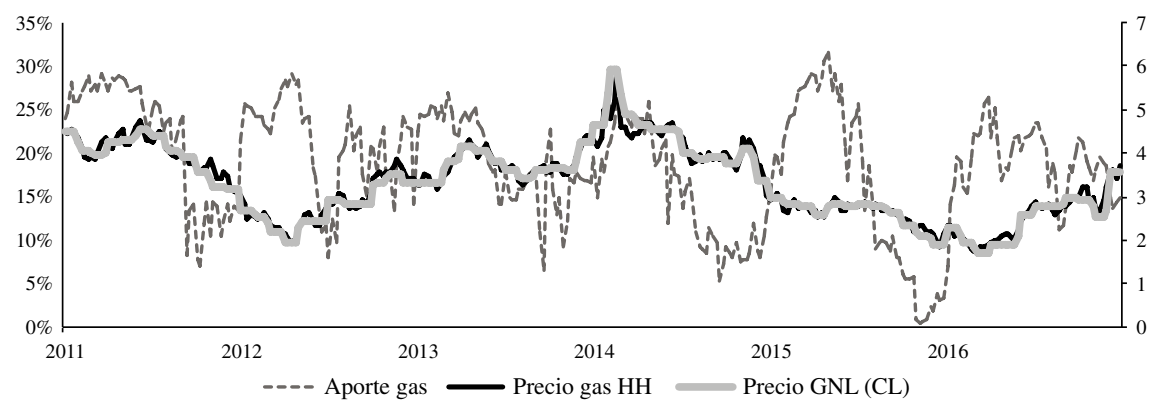

FIGURA 4

RELACION ENTRE APORTES DE CARBON Y SUS PRECIOS

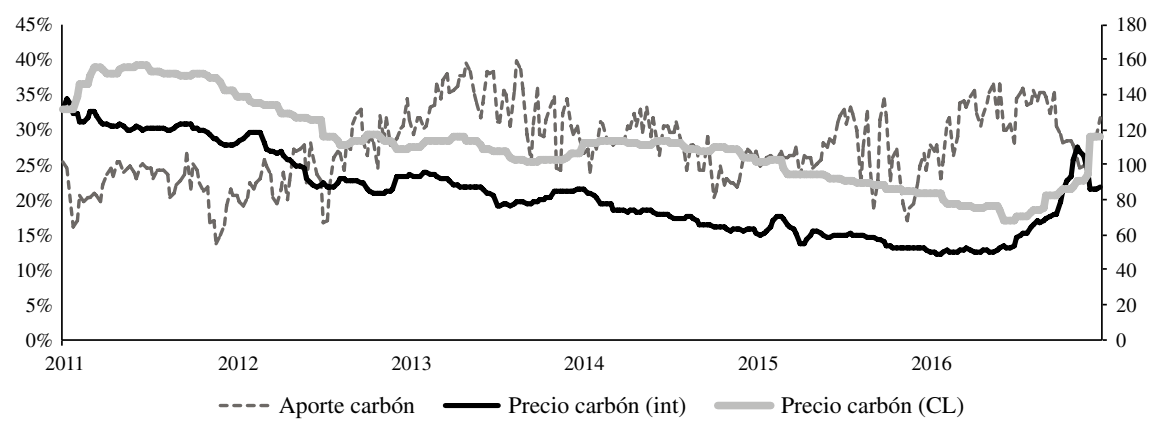

En el caso de correlación entre los precios de combustibles y el costo marginal, en la mayoría de los años es positiva, salvo en 2012 y 2016. La conclusión principal de este ejercicio es que, probablemente, los precios de combustibles que observamos no reflejan de manera precisa los costos que declaran las centrales (sea porque las plantas han suscrito contratos muy distintos de los precios internacionales o de combustibles colocados en Chile u otras razones que escapan al marco del presente análisis).

Las Figuras 3 y 4 muestran gráficamente la relación entre los precios de gas y carbón observado y los aportes de generación eléctrica de esas tecnologías. Se observa muy bien la tendencia hacia la baja en los precios, pero no siempre esto se refleja de manera clara en los mencionados aportes. 
Evidentemente el análisis de correlaciones no es lo suficientemente profundo para concluir acerca de las causalidades entre estas series de tiempo, además en el modelo chileno hay que tener en cuenta el efecto de la generación hidroeléctrica. Otro problema para usar los precios de combustibles es el desconocimiento de la "velocidad" (número de períodos) con la que los precios internacionales comienzan a reflejarse en los contratos chilenos.

En definitiva, en el modelo VAR que hemos escogido se ha optado por usar los aportes de generación térmica y no los precios de combustibles por las siguientes tres razones:

1. A diferencia de los precios de referencia o referenciales, los aportes deberían reflejar los costos reales que tienen las empresas generadoras en cuanto a los combustibles.

2. En el modelo VAR, este enfoque permite modelar los aportes de gas y carbón en función de generación hidroeléctrica y el estado de embalses, lo que sería incorrecto si fuera con los precios de combustibles, ya que estos se determinan en los mercados internacionales y no se ven influenciados por las características del mercado eléctrico chileno.

3. Este enfoque permite tener en cuenta la disponibilidad del combustible para generación en el caso de gas natural, factores que no se determinan por los costos de los combustibles, pero que impactan el costo marginal de manera directa.

En todo caso, los impactos de aportes en nuestro modelo pueden ser interpretados como los impactos de los precios de combustibles sobre el costo marginal, bajo el supuesto que la estructura de los costos no cambia y el costo declarado refleja el costo de combustible real ${ }^{13}$.

\section{Variables, fuentes y datos}

Las fuentes de las variables utilizadas en nuestro modelo VAR están presentados en la Tabla 2. En el presente análisis hemos optado por utilizar los datos con frecuencias semanales para evitar el problema de frecuencia alta en el modelo VAR, transformando los datos con frecuencia diaria ${ }^{14,15}$.

13 Es importante destacar que el sistema eléctrico chileno se basa en un mecanismo de costos declarados que pueden ser auditados por el Coordinador eléctrico.

14 En caso de costos marginales, se dispone de los datos horarios. Sin embargo, la alta frecuencia no es ventajosa en el modelo VAR, ya que, probablemente, los shocks en características del sistema eléctrico necesitan tiempo para transmitirse al precio. Para ello, llevamos los datos horarios a diarios ponderado por generación.

15 Con excepción de los precios de combustibles internacionales en los fines de semana y festivos cuando no se hace el comercio. En estos casos se ha usado el valor del día anterior. En todos estos casos calculamos un promedio simple semanal sin ponderaciones para todas las variables del modelo. 
TABLA 2

DESCRIPCION DE VARIABLES RELEVANTES PARA EL MODELO

\begin{tabular}{|l|l|l|l|}
\hline \multicolumn{1}{|c|}{ Tema } & \multicolumn{1}{c|}{ Variable } & \multicolumn{1}{|c|}{ Descripción } & \multicolumn{1}{c|}{ Fuente } \\
\hline $\begin{array}{l}\text { Precio } \\
\text { energía }\end{array}$ & $X_{0 t}-\mathrm{CMG}_{t}$ & $\begin{array}{l}\text { Costos marginales, USD/MWh. } \\
\text { Datos horarios llevados a semanales. } \\
\text { Los datos fueron ponderados a base de } \\
\text { la generación horaria del SIC. }\end{array}$ & CMg - CDEC-SIC \\
\hline $\begin{array}{l}\text { Aportes } \\
\text { diarios }\end{array}$ & $\begin{array}{l}X_{1 t}-\mathrm{GAS}_{t} \\
X_{2 t}-\mathrm{CARBON}_{t} \\
X_{3 t}-\mathrm{HIDRO} t\end{array}$ & $\begin{array}{l}\text { Energía generada por centrales } \\
\text { hidroeléctricas, GWh } \\
\text { Energía generada por centrales } \\
\text { renovables no convencionales variables } \\
\text { (solar y eólica), GWh. }\end{array}$ & $\begin{array}{l}\text { Superintendencia } \\
\text { de Electricidad y } \\
\text { Combustibles (SEC) }\end{array}$ \\
\hline Embalses & $X_{4 t}-$ EMBALSE $_{t}$ & $\begin{array}{l}\text { Energía embalsada en los embalses del } \\
\text { SIC, GWh. }\end{array}$ & SEC \\
\hline
\end{tabular}

Nuestro análisis se centra entre el 2011 y 2016, con los datos semanales, tenemos 281 observaciones para el modelo VAR. La Tabla 3 muestra las estadísticas descriptivas.

TABLA 3

ESTADISTICAS DESCRIPTIVAS

\begin{tabular}{|l|r|r|r|r|r|}
\hline \multicolumn{1}{|c|}{ Variable } & Obs. & Promedio & $\begin{array}{r}\text { Desv. } \\
\text { estándar }\end{array}$ & Mínima & Máxima \\
\hline CMg Diego de Almagro (DA) & 312 & 139,53 & 77,83 & 20,02 & 282,71 \\
CMg Cardones (CA) & 312 & 133,48 & 71,41 & 19,89 & 263,23 \\
CMg Quillota (QU) & 312 & 134,93 & 66,54 & 29,67 & 299,76 \\
CMg Alto Jahuel (AJ) & 312 & 141,06 & 72,08 & 29,22 & 314,60 \\
CMg Charrúa (CH) & 312 & 129,26 & 69,71 & 22,98 & 302,87 \\
CMg Puerto Montt (PM) & 312 & 138,23 & 70,32 & 25,30 & 303,84 \\
Aporte gas & 312 & 0,18 & 0,06 & 0,01 & 0,31 \\
Aporte carbón & 312 & 0,27 & 0,05 & 0,13 & 0,39 \\
Aporte hidro & 312 & 0,41 & 0,10 & 0,22 & 0,70 \\
Embalse & 312 & $1.840,71$ & 861,80 & 130,10 & $3.845,83$ \\
\hline
\end{tabular}

\section{La dispersión geográfica de los costos marginales}

Las Figuras 5A y 5B comparan gráficamente los costos marginales entre las barras del Norte (Diego de Almagro), Centro (Quillota) y Sur (Puerto Montt), tomando Quillota como referencia, debido a su posición geográfica y su importancia en términos de demanda. 


\section{FIGURA 5A}

EVOLUCION DE COSTOS MARGINALES: NORTE-CENTRO, USD/MWH

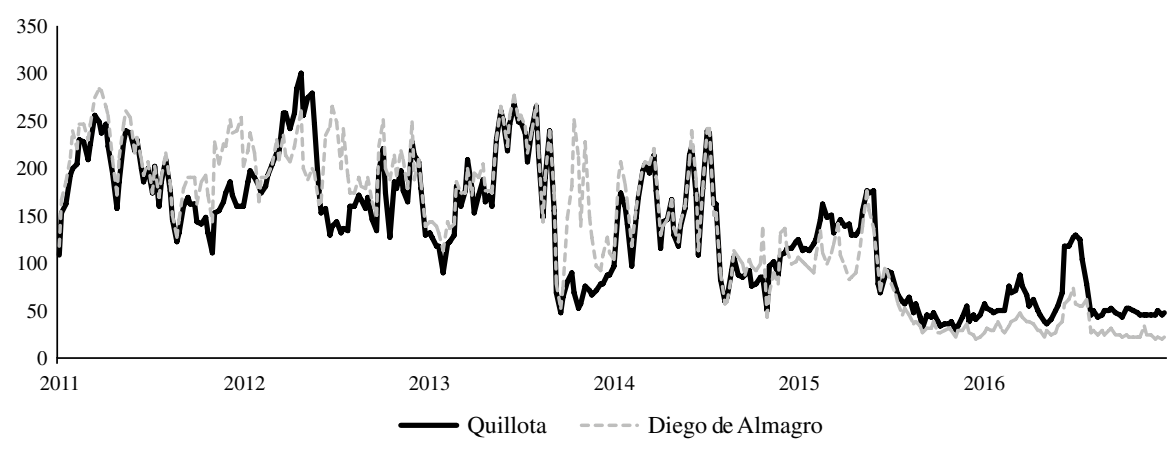

FIGURA 5B

EVOLUCION DE COSTOS MARGINALES: CENTRO-SUR, USD/MWH ${ }^{16}$

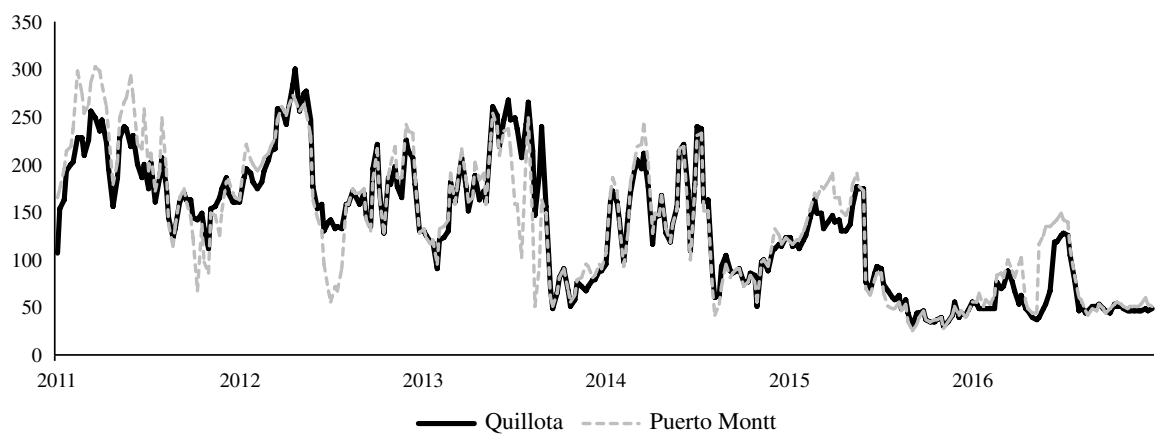

En el Norte hasta 2014 el costo marginal era más alto que en Quillota, revirtiendo esa tendencia desde 2015. En cambio, en el Sur, antes de 2014 el costo marginal era sistemáticamente más alto que en Quillota, haciéndose más estable últimamente.

16 Las diferencias entre barras pueden deberse a diferencias relevantes entre oferta y demanda alrededor de cada barra así como a congestión en las redes de transmisión. 


\section{FIGURA 6}

GRAFICO APORTES GAS, CARBON, HIDROELECTRICIDAD Y ENERGIA EMBALSADA

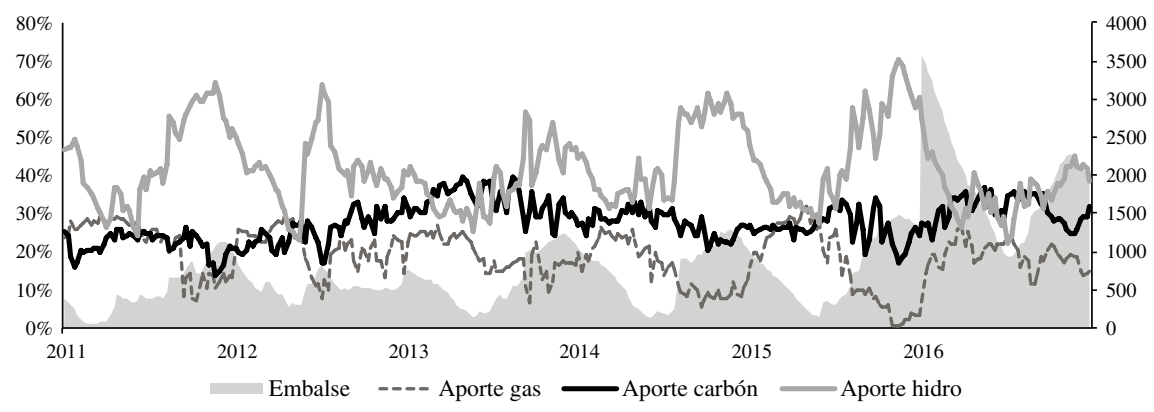

\section{Otras variables del modelo}

La Figura 6 muestra la evolución del resto de las variables consideradas en el modelo principal. La energía embalsada tiene el carácter estacional, lo que es natural para un país como Chile en cuanto a condiciones climáticas. Los aportes de hidroelectricidad también siguen esta estacionalidad, mientras que los aportes de gas y carbón compensan de manera conjunta la deficiencia de la generación hidroeléctrica cuando esta ocurre.

\section{VAR estructural}

Podría ser razonable imponer algunas restricciones lógicas a nuestro modelo de VAR. Por ejemplo, el nivel del embalse se determina por las condiciones meteorológicas, y no es razonable permitir que el nivel del embalse dependa de forma directa de aportes de gas natural o del costo marginal.

Después de estimar el VAR simple con 3 rezagos, se realizó el test de casualidad de Granger -la prueba estadística de casualidad en cada ecuación del modelo VAR ${ }^{17}$. Debido a los resultados de este testeo y desde la lógica del funcionamiento del mercado, se optó por utilizar un VAR estructural que se describe a continuación. Esta forma funcional permite probar empíricamente cuáles factores son más determinantes de la evolución del costo marginal.

A partir del análisis económico del sector y el funcionamiento efectivo del mercado eléctrico chileno, se plantean las siguientes relaciones estructurales entre las variables del modelo:

17 Los detalles del test de Granger para todas las variables del modelo principal se presentan en el Anexo 1C. 
- La variable de interés, el costo marginal, se relaciona con todas las variables del modelo, ya que estas representan sus principales determinantes.

- Los aportes de gas en nuestro modelo no se relacionan con el costo marginal, ya que la causalidad va en la dirección contraria (aportes de gas determinan el costo marginal). Además, se relaciona con la energía embalsada solo por medio de generación hidroeléctrica.

- Los aportes de carbón tampoco se relacionan con el costo marginal, ya que la causalidad va en la dirección contraria (aportes de carbón pueden determinar el costo marginal). Además, no se relaciona con aportes de gas (ya que el carbón se despacha primero), ni con la energía embalsada de forma directa, solo mediante generación hidroeléctrica.

- Los aportes hidro se modelaron en función de la generación hidroeléctrica, estado de embalses y costo marginal de la barra explicada en el modelo. Lo último, porque el despacho hidroeléctrico depende del costo de oportunidad de almacenar agua ${ }^{18}$.

- La energía embalsada solo se relaciona con los rezagos de la misma variable, ya que es determinada por condiciones climáticas y no por condiciones del sector eléctrico.

En la forma matemática, el vector de variables junto con la estructura lógica anteriormente mencionada queda evidenciado en al siguiente matriz de restricciones.

$$
X_{T}=\left[\begin{array}{c}
X_{0 t} \\
X_{1 t} \\
X_{2 t} \\
X_{3 t} \\
X_{4 t}
\end{array}\right]=\left[\begin{array}{c}
\text { CMg }_{t} \\
\text { AporteGas }_{t} \\
\text { AporteCarbon }_{t} \\
\text { AporteHidro }_{t} \\
\text { EstadoEmbalse }_{t}
\end{array}\right] S=\left[\begin{array}{ccccc}
\alpha_{0,0} & \alpha_{0,1} & \alpha_{0,2} & \alpha_{0,3} & \alpha_{0,4} \\
0 & \alpha_{1,1} & \alpha_{1,2} & \alpha_{1,3} & 0 \\
0 & 0 & \alpha_{2,2} & \alpha_{2,3} & 0 \\
\alpha_{3,0} & 0 & 0 & \alpha_{3,3} & \alpha_{3,4} \\
0 & 0 & 0 & 0 & \alpha_{4,4}
\end{array}\right]
$$

Por dificultades de interpretación y la cantidad de información y como es habitual para los modelos VAR, omitimos la presentación de los resultados numéricos del modelo y nos concentraremos en los resultados de descomposición de varianza del costo marginal.

18 Se realizó en análisis de sensibilidad del modelo distinguiendo entre esas dos tecnologías, sin alteración significativa de los resultados, ver Anexo $2 \mathrm{~A}$. 


\section{RESULTADOS}

En esta sección inspeccionamos los resultados, así como la precisión de nuestro modelo VAR estructural para cada una de las seis barras consideradas y también presentamos los resultados de la descomposición de la varianza del costo marginal eléctrico acorde con las variables que este modelo componen, es decir, aportes de gas, carbón e hidroelectricidad, estado de embalses y propio rezago del costo marginal en la misma barra.

\section{Modelo VAR}

La Figura 7 muestra seis gráficos, cada uno de ellos corresponde a una barra estudiada y muestra el costo marginal real observado (gris oscuro) y el costo marginal que nuestro modelo VAR predice (gris claro). Los valores predichos corresponden a las estimaciones simples, donde el costo marginal en un período se estima de acuerdo con los coeficientes del modelo y los valores pasados de las variables explicativas.

Como se puede observar, el modelo explica el proceso de determinación del costo marginal de forma bastante precisa, se respetan las tendencias generales y en muchos casos las fluctuaciones puntuales y temporales. Con esto concluimos que, en general, el VAR estructural que especificamos en la sección anterior se ajusta bien a la realidad del mercado eléctrico y proporciona las proyecciones acertadas del costo marginal.

\section{Descomposición de varianza}

La descomposición de la varianza permite tener una aproximación de la importancia relativa que tienen los shocks en las variables del sistema, separando la variación en el error de pronóstico de esta última entre los distintos componentes de innovaciones. Así, basados en datos históricos es posible visualizar la evolución a lo largo del tiempo de la importancia promedio de cada variable del sistema para explicar las variaciones del costo marginal.

La Figura 8 muestra la descomposición del costo marginal eléctrico en la barra Quillota (a modo de ejemplo) a lo largo de 15 períodos. Para todas las variables, la estimación de la parte de varianza que se debe a un shock se estabiliza después de 3-4 períodos y se mantiene durante el resto del tiempo. En todos los casos, las estimaciones son estadísticamente significativas a $95 \%$.

La Tabla 4 resume el mismo resultado para todas las barras, solo concentrándose en el valor del período 15, último período considerado para esta estimación. 


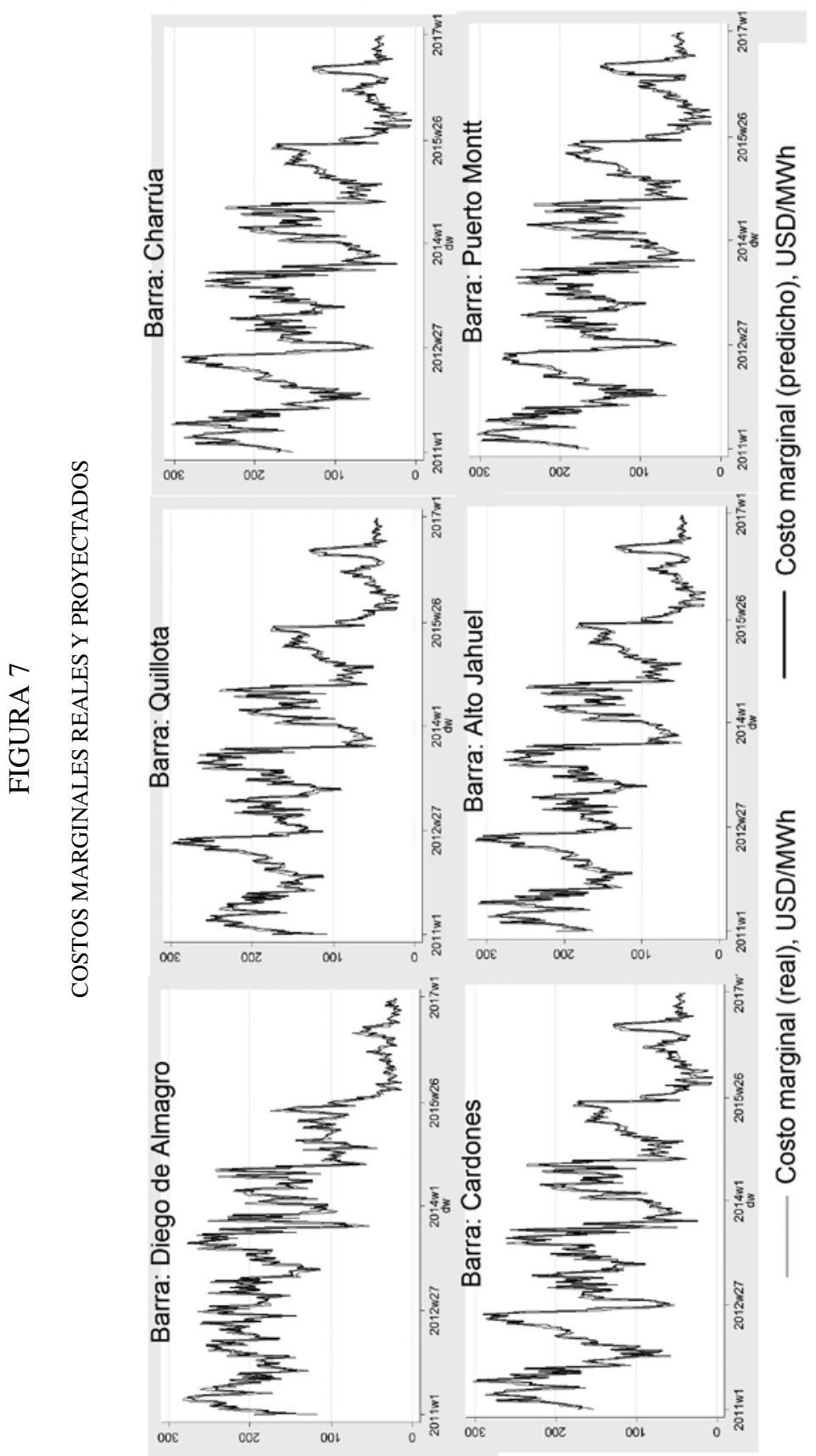




\section{FIGURA 8}

\section{DESCOMPOSICION DE LA VARIANZA DEL COSTO MARGINAL (QUILLOTA)}
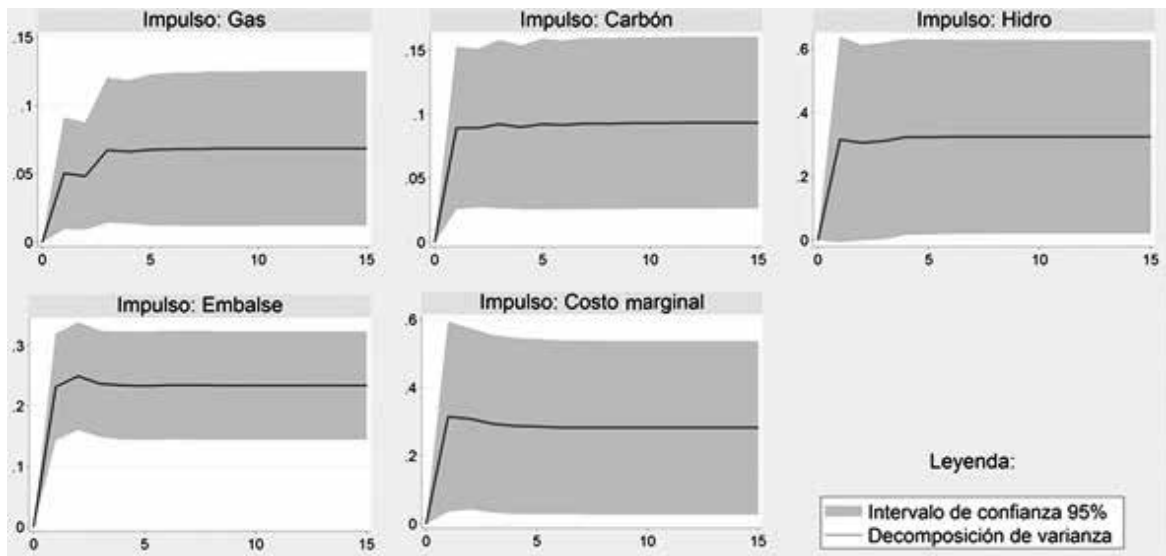

Leyenda:

Intervalo de confianza $95 \%$

Decomposición de varianza

TABLA 4

DESCOMPOSICION DE VARIANZA DEL COSTO MARGINAL, PERIODO 15

\begin{tabular}{|l|c|c|c|c|c|c|}
\hline Shock & $\begin{array}{c}\text { Diego de } \\
\text { Almagro } \\
(\mathrm{DA}) \\
(\%)\end{array}$ & $\begin{array}{c}\text { Cardones } \\
(\mathrm{CA}) \\
(\%)\end{array}$ & $\begin{array}{c}\text { Quillota } \\
(\mathrm{QU}) \\
(\%)\end{array}$ & $\begin{array}{c}\text { Alto } \\
\text { Jahuel } \\
(\mathrm{AJ}) \\
(\%)\end{array}$ & $\begin{array}{c}\text { Charrúa } \\
(\mathrm{CH}) \\
(\%)\end{array}$ & $\begin{array}{c}\text { Puerto } \\
\text { Montt } \\
(\mathrm{PM}) \\
(\%)\end{array}$ \\
\hline Aporte gas & 9,1 & 8,5 & 7,3 & 7,8 & 7,6 & 6,3 \\
Aporte carbón & 10,7 & 11,4 & 9,5 & 10,5 & 10,3 & 7,7 \\
Aporte hidro & 28,0 & 27,6 & 35,9 & 36,6 & 40,7 & 47,0 \\
Embalse & 7,6 & 7,5 & 17,3 & 17,4 & 16,9 & 16,2 \\
Costo marginal & 44,5 & 45,0 & 29,9 & 27,6 & 24,5 & 22,8 \\
\hline
\end{tabular}

Para todas las barras consideradas se observa que una parte importante de la varianza en el costo marginal está explicado por innovaciones en las variables relacionadas con disponibilidad de agua: generación hidroeléctrica y el estado de los embalses. Este resultado es lógico para el Sistema Interconectado Central debido a su composición y funcionamiento, donde el rol de los combustibles es considerable, pero no primario.

Otro punto importante es que para todas las barras de $25 \%$ a $50 \%$ de la variación del costo marginal están asociados con la tendencia en la misma barra. Esto quiere decir que una parte importante de la variación del costo marginal no se puede atribuir a las innovaciones en los factores que especifica el modelo, sino a las innovaciones 
en el costo marginal propiamente tal: del sistema o de la propia barra. Es decir, este resultado indica que probablemente existen otros factores que este modelo no captura. En la siguiente sección discutimos este punto más en detalle.

\section{Modelo de corrección de errores simple}

Como una manera alternativa de estimar los determinantes del costo marginal en el período bajo estudio se buscó una herramienta complementaria. Un modelo de corrección de errores simple permite analizar la disminución del costo marginal desde el punto de vista de relaciones de largo plazo entre el costo marginal y sus determinantes, descrito en la siguiente ecuación econométrica:

$$
\Delta x_{t}=\beta_{0}+\beta_{1} \Delta x_{t-1}+\beta_{2} \Delta x_{t-2}+\beta_{3} \Delta x_{t-3}+\gamma\left(x_{t}-y_{t}\right)+e_{t}
$$

En donde $\boldsymbol{x}$ corresponde al costo marginal, $\boldsymbol{y}$ corresponde al determinante del mismo. El error aquí se compone de dos partes: por una parte el error habitual $\boldsymbol{e}$, y por otra parte la relación de largo plazo entre la variable de interés y su determinante.

Si bien este enfoque permite el análisis intuitivo de la relación entre el costo marginal y sus determinantes, es de alcance limitado porque no permite la relación entre los determinantes y solo se concentra en las relaciones bilaterales.

Los resultados se encuentran en la Tabla 3A del Anexo para 6 modelos de corrección del error simple. De estos resultados podemos concluir los mensajes similares a los resultados de nuestro VAR. Primero, ninguno de los determinantes del modelo explica muy bien la tendencia de los costos marginales -son los rezagos de la misma variable que aportan gran parte de la explicación-. Segundo, la tendencia hacia la baja de los costos marginales es más notable en el Norte en comparación con el Sur (para las barras del Norte, todos los rezagos tienen los coeficientes negativos, mientras en el Sur, solo dos). Y tercero, el impacto de los factores relacionados con la disponibilidad de agua para generación eléctrica es más notoria en el Sur.

\section{DISCUSION}

Después de haber analizado la relación estadística entre el costo marginal de generación eléctrica y las características del sistema eléctrico chileno, que se han utilizado para explicar su comportamiento, hemos calculado la descomposición de varianza del costo marginal. En esta sección analizamos y presentamos la interpretación de estos resultados, distinguiendo entre tres grupos de factores: categoría "Agua" agrupa efectos de embalses y generación hidroeléctrica; categoría "Combustibles", efectos de gas y carbón, y categoría "Otros", el resto de los factores.

Este análisis representa una mirada más agregada de la información presentada en Tabla 5. La Figura 9 muestra la descomposición de varianza agregada a lo largo 


\section{FIGURA 9}

\section{DESCOMPOSICION DE LA VARIANZA DEL COSTO MARGINAL ENTRE TRES FACTORES, 2011-16}

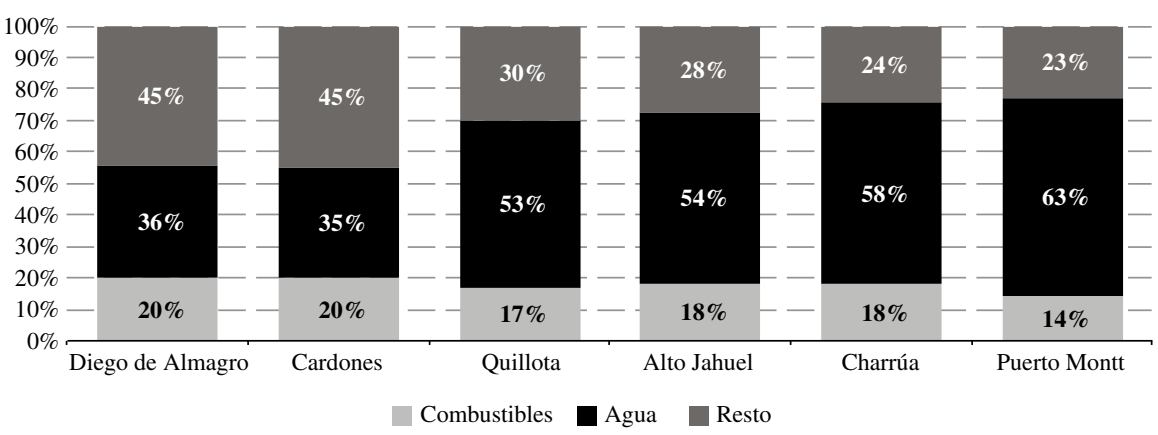

del SIC, de Norte al Sur: Diego de Almagro (DA), Cardones (CA), Quillota (QU), Alto Jahuel (AJ), Charrúa (CH), Puerto Montt (PM).

\section{Agua y combustibles}

El impacto de la categoría "Agua" es muy pronunciado en todas las barras consideradas a lo largo del país. Sin embargo, la importancia de los efectos relacionados con hidroelectricidad aumenta considerablemente hacia el Sur: si en las barras del Norte el agua explica 34\%-35\% de la variación del costo marginal eléctrico, este número aumenta hasta 56\% en el Centro, y hasta 58\%-60\% en el Sur. Este patrón de la importancia de agua es lógico, a causa de la geografía del SIC, porque las grandes centrales hidroeléctricas no están situadas en el Norte, sino en el Centro y, en su mayoría, en el Sur del país (Tabla 5).

El impacto de la categoría "Combustibles" varía entre $21 \%$ para las barras del Norte y 16\%-18\% para las barras del Centro y Sur. Como muestra la Figura 9, el efecto de las fuentes termoeléctricas es similar entre las diferentes zonas a diferencia del efecto del agua, lo que refleja, también, la distribución de las plantas termoeléctricas de manera más homogénea geográficamente (Tabla 5).

Históricamente, estos dos factores, el agua y los combustibles, se identificaban como los únicos determinantes del costo marginal (Bustos y Fernández, 2013). No obstante, esto no parece ser cierto en el período bajo análisis. De la Figura 9 llama la atención que la categoría "Resto", es decir, el conjunto de otros factores, tiene más importancia que la categoría "Combustibles". En vista de los resultados de este análisis y el progreso del sector eléctrico, hay un conjunto de otros factores que son muy relevantes al ser considerados en conjunto. 


\section{TABLA 5}

\section{CAPACIDAD INSTALADA POR ZONA (SIC) ${ }^{19}$}

\begin{tabular}{|l|c|c|r|r|r|c|}
\hline \multirow{2}{*}{ Zona } & \multicolumn{2}{|c|}{ Termo } & \multicolumn{2}{c|}{ Hidro } & \multicolumn{2}{c|}{ ERNC (solar + eólica) } \\
\cline { 2 - 7 } & MW & Unidades & MW & Unidades & MW & Unidades \\
\hline Norte & 1.811 & 27 & 33 & 4 & 2.017 & 52 \\
Centro & 5.887 & 74 & 6.099 & 108 & 250 & 20 \\
Sur & 287 & 31 & 507 & 51 & 189 & 3 \\
\hline
\end{tabular}

Fuente: CNE, capacidad de generación instalada.

Es factible mencionar que la importancia de la categoría "Resto" también difiere entre las zonas geográficas. A continuación exploramos los posibles factores que forman parte de la categoría "Resto", así como las razones de las divergencias entre las zonas.

\section{Otros factores}

En vista de las recientes tendencias en el sector eléctrico chileno, hay algunas variables que el modelo no captura, pero que podrían haber jugado un rol importante en la reciente caída del costo marginal en el SIC o en algunas zonas geográficas del mismo.

En primer lugar, el aumento en la generación de ERNC con el costo de generación cercano a cero y el reemplazo de la generación a diésel con costo de generación alto, podría ser uno de los factores principales.

El segundo lugar, podría existir un efecto de restricción de transmisión que se podría manifestar en las barras donde no hay suficiente capacidad de transmisión para evacuar toda la generación a bajo costo a los lugares de consumo. Esto podría explicar diferencias geográficas en la variabilidad de los costos marginales ${ }^{20}$.

Por último, si la demanda eléctrica ha crecido a una tasa menor que la capacidad de generación, podríamos observar un costo marginal a la baja igualmente ${ }^{21}$.

La Figura 10 muestra las series de generación a base de diésel y ERNC ${ }^{22}$ desde el año 2011 hasta el segundo semestre de 2016. En los últimos 3 años la generación ERNC ha aumentado: en 2016 el aporte ERNC es más de 12 veces mayor que antes

19 En SIC consideramos la siguiente terminología. Norte: regiones II, III y IV; Centro: regiones V, VI, VII, VIII y XIII; Sur: regiones IX, X y XIV.

20 El enfoque del análisis y los resultados del modelo incorporando el efecto de transmisión están presentados en el Anexo 2B.

21 Se realizó un análisis de sensibilidad del modelo incorporando el efecto de la demanda, sin alteración significativa de los resultados. Para mayores detalles ver Anexo 2C.

22 Para enfatizar intermitencia, aquí ERNC incluye solamente solar y eólica, excluyendo minihidro y biomasa. 
FIGURA 10

GENERACION DIARIA POR TECNOLOGIA, GWH - ERNC Y DIESEL

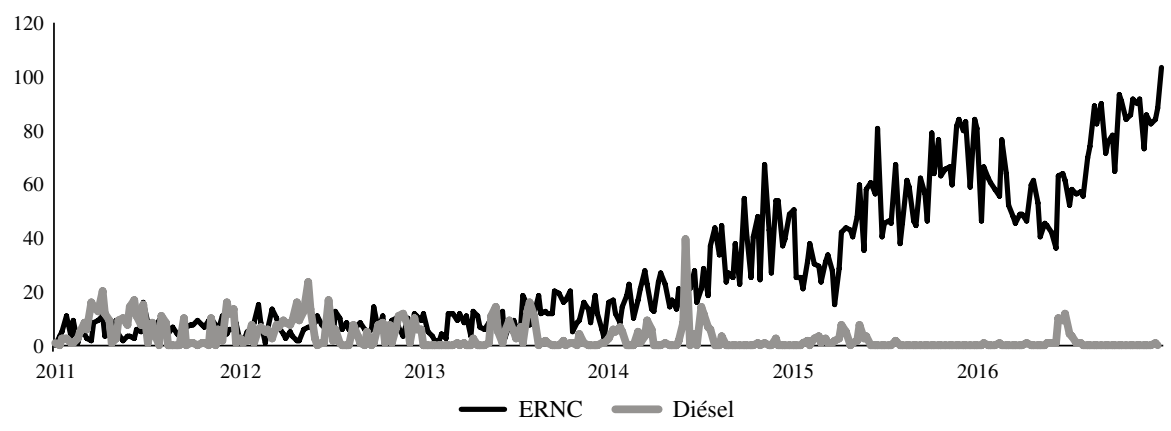

de 2014. A su vez, la generación diésel perdió su importancia dramáticamente, siendo reemplazado por otras tecnologías: en el 2016 el aporte de esta tecnología es 45 veces menor, en promedio, que antes de 2014.

En virtud de la naturaleza de estas tecnologías - despacho en hora punta para diésel e intermitencia debido a condiciones naturales para tecnologías ERNC-, estadísticamente sus aportes tienen volatilidades altas, como muestra la Figura 10. Estas volatilidades son distintas de las volatilidades de las variables de nuestro modelo VAR (los aportes de gas, carbón o hidroelectricidad).

La Figura 11 compara los coeficientes de variación ${ }^{23}$ para las variables de nuestro modelo VAR y aportes de diésel y ERNC, usando datos semanales y datos diarios. Los coeficientes de variación son muy consistentes entre dos frecuencias para todas las variables del modelo VAR (aportes de gas, carbón, hidroelectricidad y estado de embalses). Sin embargo, no es el caso para aportes de diésel o para aportes de tecnologías ERNC.

Además de ser mayor que para el resto de las variables, la variable aporte de diésel muestra los coeficientes de variación no consistentes en el tiempo: si antes del 2016 el coeficiente de variación era mayor para los datos semanales, en 2016 esto se revirtió. En otras palabras, si antes de 2016 el aporte de diésel variaba entre semanas, ahora varía más entre los días -debido a su tendencia de uso decreciente en el tiempo-.

En cuanto a los aportes ERNC, el coeficiente de variación diario es mayor, y además, se observa clara tendencia hacia la baja en el tiempo. Esto también se debe

23 El coeficiente de variación se usa en estadística cuando se desea hacer referencia a la variabilidad de la variable en una escala neutra, es decir, sin relación con el tamaño de la media. Su fórmula expresa la desviación estándar como porcentaje de la media aritmética, mostrando una mejor interpretación porcentual del grado de variabilidad que la desviación típica o estándar. 


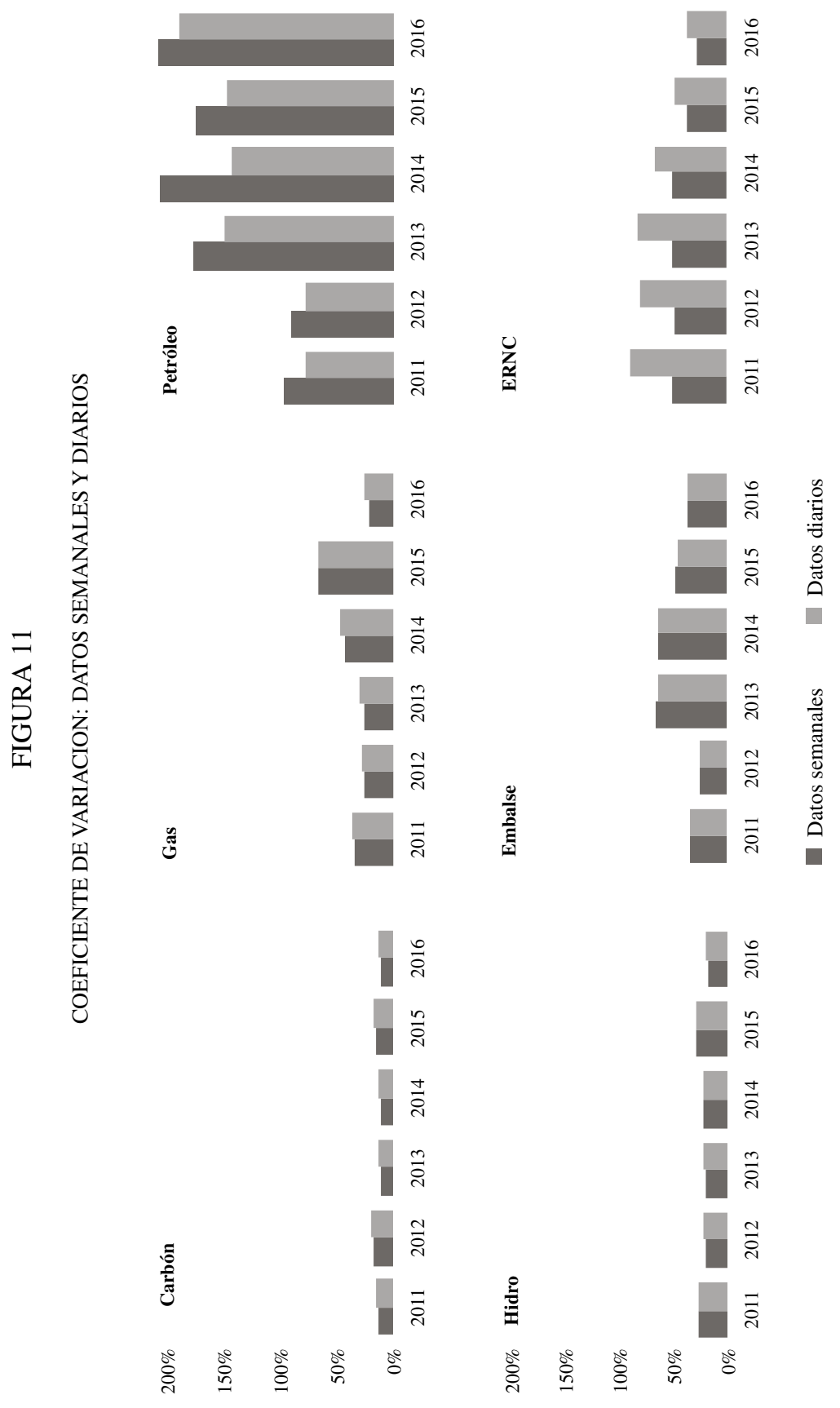


a la intermitencia de las fuentes ERNC -si al nivel semanal, en promedio, ERNC genera la cantidad de energía parecida, esas cantidades pueden diferir mucho entre los días de la semana, ya que están sujetos a las condiciones climáticas-.

Tomando en cuenta estos patrones de volatilidad, estadísticamente es más preciso usar los datos diarios para capturar los efectos de estas variables en el costo marginal. Además, a causa de las tendencias de sus aportes en el tiempo, es mejor no incorporar estas variables en nuestro modelo VAR, ya que la importancia de estos factores está cambiando en el tiempo, haciendo los efectos en el costo marginal y su importancia relativa también diferentes en el tiempo.

De todas maneras, es interesante cuantificar los impactos de ERNC y diésel en el costo marginal de manera ilustrativa. Para ello estimamos modelos VAR simplificados, donde en el modelo A el costo marginal depende de su propio rezago y de los aportes ERNC, mientras ERNC sigue un proceso independiente; y en el modelo B, el costo marginal depende de su propio rezago y aportes de diésel, mientras la variable aportes diésel sigue un proceso independiente ${ }^{24}$.

Estos modelos se estiman sobre la base de datos diarios, para así capturar mejor la intermitencia de esas dos fuentes de generación sin perder la significatividad estadística. Después de estimar el modelo, calculamos el porcentaje de varianza de costo marginal explicado por cada variable.

\section{Aportes ERNC}

La Figura 12 se basa en el modelo simplificado A, y muestra la importancia relativa de los aportes ERNC en el costo marginal, haciendo distinción entre dos períodos de análisis: años 2011-2013 y años 2014-2016, para las seis barras consideradas.

Hay dos mensajes claros de esta figura: primero, la importancia de generación ERNC para el costo marginal ha aumentado más del doble en 2014-2016 en comparación con el período anterior; segundo, este impacto es significativamente mayor para las barras de la zona Norte, donde la mayoría de las plantas ERNC están situadas geográficamente (Tabla 5). Además de la importancia para explicar la categoría "Resto" de la Figura 9, este análisis aporta en explicar las diferencias entre las barras ${ }^{25}$.

Sin embargo, parece ser que aportes de ERNC no es la única razón de diferencias entre las barras en cuanto a otros factores que expliquen el costo marginal. Si fuera así, ERNC sería más relevante para la barra Diego de Almagro que para la barra Cardones. Este resultado hace pensar que hay otros factores que pueden explicar

24 También se ha construido el modelo C, donde el costo marginal depende de su propio rezago y de los aportes ERNC y aportes de diésel, mientras ERNC sigue un proceso independiente y aportes de diésel dependen de su propio rezago y de los aportes ERNC. Este modelo permite comparar las importancias relativas de los aportes ERNC y de diésel. Las conclusiones son muy similares a los modelos A y B.

25 Otra manera de ver lo mismo, es considerar el vertimiento solar. En el 2014 y 2016 hay vertimiento en dos barras del Norte, Diego de Almagro y Cardones, siendo Diego de Almagro la barra que alcanza $24 \%$, un porcentaje extremadamente alto de costos marginales bajos debido a cantidad de energía solar en 2016. 


\section{FIGURA 12}

\section{DESCOMPOSICION DE LA VARIANZA DEL COSTO MARGINAL: IMPACTO ERNC, MODELO A}

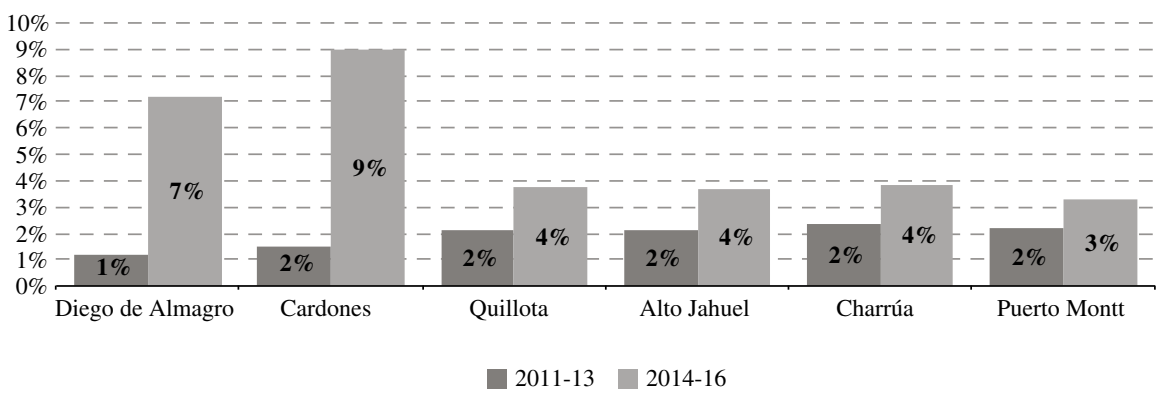

las diferencias no explicadas por agua y combustibles entre las zonas geográficas, posiblemente relacionados con el sistema de transmisión que conecte las barras del Norte con el Centro y el Sur.

\section{Aportes diésel}

La Figura 13 se basa en el modelo simplificado B, y muestra la importancia relativa de los aportes de diésel en el costo marginal, haciendo distinción entre dos períodos de análisis: años 2011-2013 y años 2014-2016, para las seis barras consideradas.

La caída en despacho de las centrales a diésel tuvo un efecto pronunciado en los costos marginales -el hecho de que hay suficiente capacidad más barata para cubrir la demanda punta ha bajado el costo marginal-. La importancia de los aportes de diésel ha bajado en todo el SIC, siendo el efecto más grande en el Centro: de $22 \%-26 \%$ en 2011-2013 a 15\%-16\% en 2014-2016.

Así como en el caso de ERNC, el impacto es diferente entre las barras: aportes diésel parecen ser mucho más relevantes en el Centro y Sur, en comparación con las barras del Norte.

\section{Otros modelos ${ }^{26}$}

Para poder comparar las importancias de aportes ERNC y diésel en el costo marginal, se ha desarrollado el modelo $\mathrm{C}$. Los resultados muestran que en el modelo conjunto, la importancia relativa de los factores se parece bastante a los modelos individuales,

26 Los resultados de esos modelos están presentados en los Anexos 2C y $2 \mathrm{D}$. 


\section{FIGURA 13}

\section{DESCOMPOSICION DE LA VARIANZA DEL COSTO MARGINAL: IMPACTO PETROLEO, MODELO B}

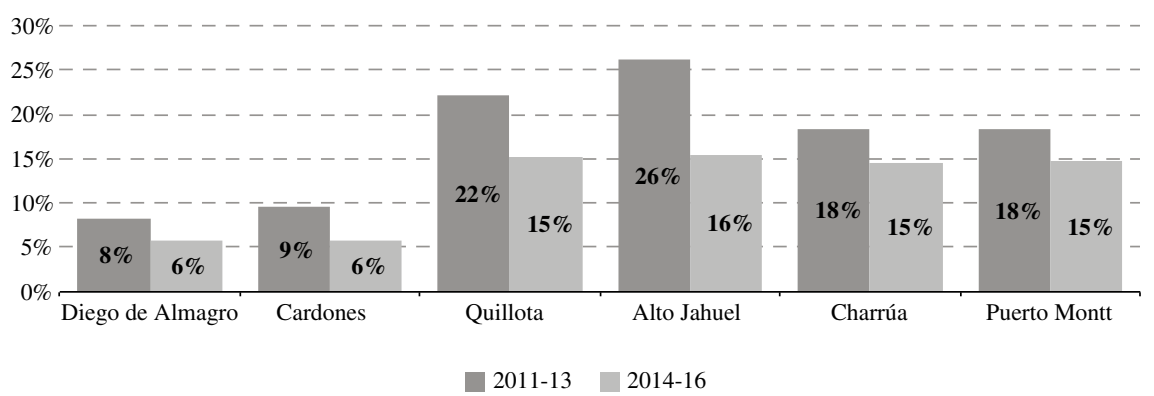

concluyendo que las dos variables son de alta importancia y son complementarias, pero en términos relativos, el fin del despacho de diésel juega un rol más importante que el aumento de la generación de ERNC.

También se ha elaborado un modelo simple con la demanda eléctrica, identificando que esa variable tiene mayor relevancia en el Centro y Sur del país, pero sin muchos cambios en el tiempo.

Por último, se ha elaborado un modelo que permite incorporar el efecto de restricciones de transmisión, encontrando que en las barras del Norte alrededor de $50 \%$ del "Resto de los factores" se puede atribuir a este factor, pero que al nivel del sistema, ese factor no debiese tener tanta relevancia, ya que las restricciones de transmisión bajan el costo marginal en algunas barras y suben en otras barras. De esa manera, el costo marginal en las barras del Centro y Sur podría haber bajado aún más sin restricciones de transmisión.

\section{CONCLUSIONES}

Después de haber analizado la relación estadística entre el costo marginal de generación eléctrica y las características del sistema eléctrico chileno, que se han utilizado para explicar su comportamiento, concluimos que mayor disponibilidad de agua, capturada por las variables del estado de embalses y generación hidroeléctrica, es el factor más relevante para explicar la variación del costo marginal. En esta línea, el factor de agua jugó un rol substancial en la reciente caída del costo marginal en el SIC, pero no es el único factor importante.

Ya no parece ser cierto en el período bajo análisis que el agua y los combustibles son los únicos determinantes del costo marginal. En vista de los resultados de este análisis y el progreso del sector eléctrico, hay un conjunto de otros factores que van ganando más importancia y ya son muy relevantes al ser considerados en conjunto. 
Los crecientes aportes de generación ERNC (en particular, eólica y solar), menor necesidad a recurrir a generación con diésel, el factor de demanda y otros factores (rezago del costo marginal como aproximación al trend general del sistema) explican casi un tercio de la variación del costo marginal, y en esta línea, un tercio de la caída de los últimos años. Eso permite decir que restando los efectos de mayor disponibilidad del agua y menores precios de combustibles, el costo marginal caería al menos 20 USD/MWh en los últimos dos años.

Estos resultados abren un campo de acción a la política energética. Es posible que acciones de Eficiencia Energética que apunten a reducir el crecimiento de la demanda, así como fomento a energías renovables no convencionales, se conviertan en instrumentos cada vez más relevantes a la hora de reducir precios spot de energía eléctrica en Chile. Igualmente, mejoras en la eficiencia de la operación en generación así como de la red de transmisión.

Podría argumentarse que caídas significativas en el precio spot podrían reducir la señal de precios en favor de mayor inversión en capacidad de generación. Sin embargo, producto de la necesidad de asegurar capacidad en el mercado chileno, la introducción de licitaciones por contratos de suministro de largo plazo en 2006 (y reformado en 2014), está llevando a que el precio de contratos sea la señal relevante para la decisión de inversión en el largo plazo, y no el costo marginal de corto plazo. Es posible preguntar ahora si con una tendencia a la baja en los costos marginales es necesario comenzar a considerar cambios en el sistema marginalista y en la remuneración a servicios complementarios que provean unidades de mayor costo de operación.

\section{REFERENCIAS}

ARELLANO, M.S. y P. SERRA (2010). "Long-term Contract Auctions and Market Power in Regulated Power Industries", Energy Policy 38 (4), pp. 1759-1763.

BUSTOS, J. (2015a). "Bidding behavior in Chilean electricity market", Energy Economics 51, pp. 288-299.

BUSTOS, J. (2015b). "El mercado de contratos de suministro eléctrico en Chile", en Economía y Energía, La experiencia chilena. Ediciones Universidad Alberto Hurtado. Santiago, Chile.

BUSTOS, J. y J. FERNANDEZ (2013). "Ralco is Coming: Investment Delay in the Chilean Power Market”, Núcleo de investigación Empresa, Sociedad y Tecnología, Universidad Mayor, Documento de Trabajo \#11.

CORREA, J.A. y J.J. GARCIA (2013). "Interconexión Eléctrica Colombia-Panamá: Impacto Sobre El Precio Spot En Panamá”, Documento de Trabajo Economía y Finanzas, Centro de Investigaciones Económicas y Financieras.

FABRA, N., J.P. MONTERO y M. REGUANT (2014). "La Competencia en el Mercado Eléctrico Mayorista en Chile". Informe preparado para la Fiscalía Nacional Económica, Santiago, Chile.

FERKINGSTAD, E., A. LOLAND y M. WILHELMSEN (2011). "Causal modeling and inference for electricity markets", Energy Economics 33, pp. 404-412.

FEZZI, C. (2007). "Econometric Analysis Of The Interaction Between The European Emission Trading Scheme And Energy Prices", Social Science Research Network Electronic Paper Collection.

HIGGS, H. y A.C. WORTHINGTON (2010). "Modelling Spot Prices in Deregulated Wholesale Electricity Markets: A Selected Empirical Review", Energy Studies Review 17 (1).

KILIAN, L. (2009). "Not All Oil Price Shocks Are Alike: Disentangling Demand and Supply Shocks in the Crude Oil Market", American Economic Review 99 (3), pp. 1053-1069.

KILIAN, L. (2012). "Why Agnostic Sign Restrictions Are Not Enough: Understanding The Dynamics Of Oil Market Var Models", Journal of the European Economic Association 10 (5), pp. 1166-1188. 
MJELDE, J.W. y D.A. BESSLER (2009). "Market integration among electricity markets and their major fuel source markets", Energy Economics 31 (3), pp. 482-491.

MORANDE, F. y R. SOTO (1996). "El mercado de clientes no regulados en la industria eléctrica". Programa de Postgrado en Economía ILADES/Georgetown University.

MORENO, J., R. MORENO, H. RUDNICK y S. MOCARQER (2012). "Licitaciones para el abastecimiento eléctrico de clientes regulados en Chile: Desafíos y oportunidades", Estudios Públicos, CEP Chile.

PARK, H., J.W. MJELDE y D.A. BESSLER (2006). "Price dynamics among U.S. electricity spot markets", Energy Economics 28 (1), pp. 81-101.

ROUBIK, E. y H. RUDNICK (2009). "Assessment of generators strategic behavior in long term supply contract auctions using portfolio concepts", Paper aceptado para presentación en 2009 IEEE Bucharest Power Tech Conference.

THOENES, S. (2015). "Understanding the Determinants of Electricity Prices and the Impact of the German Nuclear Moratorium in 2011”, Energy Journal 35 (4), pp. 61-78.

TOEWS, G. y A. NAUMOV (2015). "The Relationship Between Oil Price and Costs in Oil and Gas Industry", Oxford Centre for the Analysis of Resource Rich Economies, Research Paper 152.

VARAS, P. y H. RUDNICK (2014). "Empirical Studies of Competition in the Chilean Electricity Market". Working Paper, Pontificia Universidad Católica de Chile. 


\section{ANEXO 1}

\section{MODELO VAR}

\section{A. Prueba de estacionaridad}

Debido a que el modelo VAR solamente puede ser aplicado a las series estacionarias, se realizó la prueba de raíces unitarias para todas las variables. Se eligió la prueba de Dickey-Fuller Aumentado (ADF) como la prueba más común y más utilizada en la literatura. El resultado de esta prueba está presentado en la Tabla 1A.

TABLA 1A

PRUEBAS DE DICKEY-FULLER AUMENTADO: NIVELES Y DIFERENCIAS

\begin{tabular}{|l|c|c|c|c|}
\hline \multicolumn{1}{|c|}{ Variable } & $\begin{array}{c}\text { Estadística } \\
\text { nivel }\end{array}$ & P-valor & $\begin{array}{c}\text { Estadística } \\
\text { diferencia }\end{array}$ & P-valor \\
\hline CMg Diego de Almagro & $-3,171$ & 0,0217 & $-18,618$ & 0,0000 \\
CMg Cardones & $-3,322$ & 0,0139 & $-18,716$ & 0,0000 \\
CMg Quillota & $-3,231$ & 0,0183 & $-16,361$ & 0,0000 \\
CMg Alto Jahuel & $-3,257$ & 0,0169 & $-16,129$ & 0,0000 \\
CMg Charrúa & $-3,680$ & 0,0044 & $-15,995$ & 0,0000 \\
CMg Puerto Montt & $-3,626$ & 0,0053 & $-16,150$ & 0,0000 \\
Aporte gas & $-4,034$ & 0,0012 & $-20,348$ & 0,0000 \\
Aporte carbón & $-4,972$ & 0,0000 & $-19,588$ & 0,0000 \\
Aporte hidro & $-3,649$ & 0,0049 & $-16,285$ & 0,0000 \\
Embalse & $-1,551$ & 0,5080 & $-7,567$ & 0,0000 \\
\hline
\end{tabular}

Analizando las pruebas de raíces unitarias en niveles, se observa que la mayoría de las variables ya son estacionarias en niveles, la única excepción es la variable que corresponde al estado del embalse. En la siguiente etapa calculamos primeras diferencias y volvemos a aplicar la prueba de raíces unitarias. Después de este ejercicio, todas las variables son estacionarias en diferencias, indicando que estas variables corresponden al proceso I (1).

Teniendo esto en cuenta, y para simplificar las interpretaciones, vamos a estimar el modelo VAR en diferencias para todas las variables, incluyendo aquellas que eran estacionarias en niveles también.

\section{B. Número de rezagos}

Para elegir el número de rezagos a incluir en el modelo, se realizaron varias pruebas: prueba del error de predicción final (FPE), criterio de información de Akaike (AIC), 
criterio de información bayesiana de Schwarz (SBIC), y criterio de información de Hannan y Quinn. La Tabla 1B resume los resultados de este ejercicio.

TABLA 1B

PRUEBAS PARA DETERMINAR EL NUMERO DE REZAGOS

\begin{tabular}{|l|c|c|c|c|}
\hline \multicolumn{1}{|c|}{ Barra } & FPE & AIC & HQIC & SBIC \\
\hline Diego de Almagro & 4 & 4 & 2 & 1 \\
Cardones & 4 & 4 & 2 & 1 \\
Quillota & 3 & 3 & 2 & 1 \\
Alto Jahuel & 4 & 4 & 2 & 1 \\
Charrúa & 3 & 3 & 2 & 1 \\
Puerto Montt & 3 & 3 & 2 & 1 \\
\hline
\end{tabular}

En virtud de la necesidad del consenso entre las barras, hemos escogido tres rezagos, lo que la mayoría de las pruebas FPE y AIC indica. Aunque las pruebas HQIC y SBIC consistentemente ofrecen menor número de rezagos, hemos hecho la elección de mayor número con el razonamiento de dejar suficiente espacio y permitir la transmisión del efecto de algunas variables hacia el costo marginal en el tiempo, pero no demasiado, ya que la muestra es relativamente corta.

\section{C. Prueba de causalidad de Granger}

Podría ser razonable imponer algunas restricciones lógicas a nuestro modelo de VAR. A modo de ejemplo, el nivel del embalse se determina por las condiciones meteorológicas, y no es razonable para nuestro modelo permitir que el nivel del embalse dependa de forma directa de aportes de gas o de costo marginal.

Después de estimar el VAR simple con tres rezagos, se realizó el test de causalidad de Granger -la prueba estadística de causalidad en cada ecuación del modelo VAR. La tabla abajo muestra los p-valores de la prueba de causalidad de cada ecuación.

TABLA $1 \mathrm{C}$

SIGNIFICATIVIDAD CONJUNTA VALORES P, VAR SIMPLE

\begin{tabular}{|l|c|c|c|c|c|}
\hline \multicolumn{1}{|c|}{ Barra } & $\mathrm{CMg}$ & Gas & Carbón & Hidro & Embalse \\
\hline Diego de Almagro & 0,214 & 0,737 & 0,400 & 0,010 & 0,014 \\
Cardones & 0,215 & 0,648 & 0,371 & 0,006 & 0,007 \\
Quillota & 0,312 & 0,404 & 0,147 & 0,016 & 0,010 \\
Alto Jahuel & 0,196 & 0,351 & 0,163 & 0,014 & 0,010 \\
Charrúa & 0,007 & 0,786 & 0,078 & 0,016 & 0,012 \\
Puerto Montt & 0,077 & 0,802 & 0,283 & 0,016 & 0,024 \\
\hline
\end{tabular}


Si bien los modelos son distintos, en general, rechazamos la mayoría de las hipótesis nulas y concluimos que las variables del modelo en su conjunto no causan uno a otro. Las pruebas indican ausencia de causalidad donde la esperaríamos, por ejemplo, el costo marginal de barra Quillota no se ve causado por ninguna variable explicativa del modelo.

Por esta razón hacemos el ejercicio de imponer una estructura lógica a nuestro modelo VAR, imponiendo algunas restricciones a los coeficientes del modelo, basándose tanto en la evidencia estadística como en el conocimiento del mercado chileno y la lógica económica. Nos preocupamos de imponer justamente el número de restricciones necesario para identificación del modelo, y no imponer ninguna adicional. 


\section{ANEXO 2}

\section{ANALISIS DE SENSIBILIDAD}

\section{A. Hidroelectricidad de pasada y de embalse}

Debido a que el despacho de hidroeléctricas de pasada se realiza bajo la lógica distinta del despacho de hidroeléctricas de embalse, se realizó el análisis de sensibilidad separando los aportes en generación entre esas dos tecnologías.

Aunque la importancia de la generación hidroeléctrica y estados de embalses cambia en este caso, los resultados agregados (suma de todos los factores relacionados con agua) apenas cambia. La Figura 2A muestra esos resultados gráficamente.

\section{FIGURA 2A}

\section{DESCOMPOSICION DE LA VARIANZA DEL COSTO MARGINAL:} HIDROELECTRICIDAD DE PASADA Y DE EMBALSE

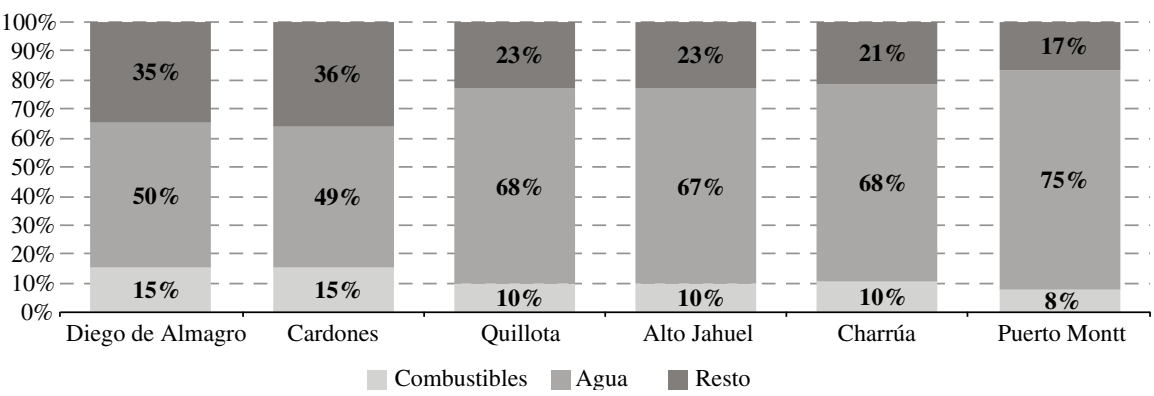

\section{B. Efecto transmisión}

Para entender si el efecto de transmisión ha sido relevante en nuestro modelo, se adoptó el enfoque de la barra de referencia: si no hay suficiente capacidad de transmisión, es probable que observemos las diferencias en los costos marginales entre la barra de interés y la barra de referencia. En nuestro caso hemos escogido la barra Quillota como la barra de referencia y la más cercana a los centros principales del consumo eléctrico en el SIC.

Incorporando una variable adicional, el costo marginal en Quillota, a nuestro modelo VAR, hace posible la distinción del resto de los factores en dos tipos: los factores comunes para todo el SIC (asociados a la barra de referencia) y los factores locales (asociados a la propia barra de interés). 
Bajo esa óptica, los factores locales podrían ser interpretados como el efecto de transmisión, en otras palabras, cuánto porcentaje de la variación del costo marginal no se explica ni por agua, ni por combustibles, ni por los otros factores comunes para el sistema eléctrico.

La Figura 2B muestra la descomposición de varianza del costo marginal a lo largo del SIC, de Norte al Sur, separando entre el efecto de transmisión (efecto idiosincrático de la propia barra) y el resto de los factores comunes para todo el sistema. Para la barra de referencia no es posible hacer esa distinción, por tanto todo se atribuye a la categoría "Resto".

\section{FIGURA 2B}

\section{EFECTO TRANSMISION EN DESCOMPOSICION DE LA VARIANZA} DEL COSTO MARGINAL, 2011-16

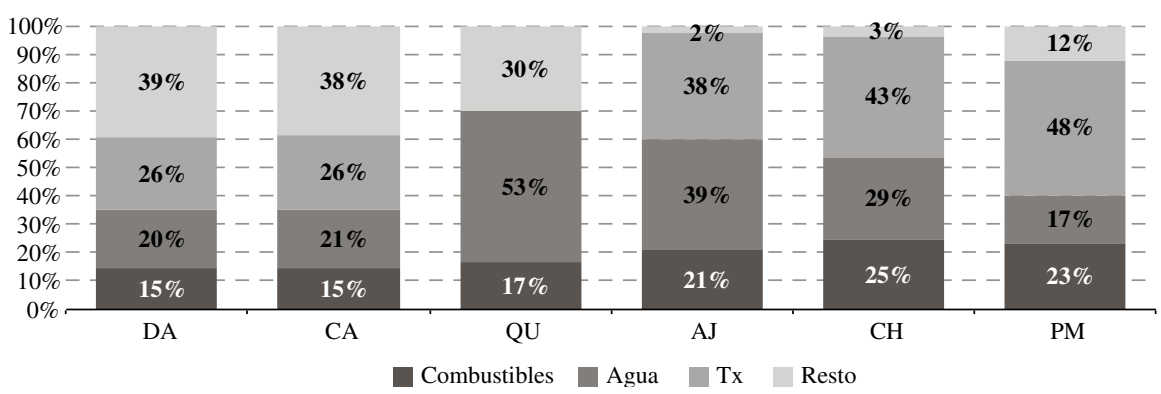

Ya que hablamos de la variación del costo marginal debido a las restricciones de transmisión, la caída fuerte en una barra (por ejemplo debido a generación de ERNC) implica que en otra barra el costo marginal no caiga tanto como podría bajo las condiciones del balanceo perfecto.

Como muestra la Figura 2B, en todas las barras el efecto de transmisión ha sido relevante, explicando alrededor de la mitad de "Otros factores", siendo mucho más relevante en las barras del Norte en términos absolutos. Así, el efecto de transmisión ayuda a explicar los otros factores en el Norte, pero no aporta razones al nivel agregado de todo el sistema eléctrico.

Dicho esto, en el presente análisis hay que tomar en cuenta que aquí la transmisión se aproxima con el costo marginal en Quillota, la medida que también puede incluir otros factores (aunque ya incluidos, como combustibles, agua, etc.).

\section{C. Demanda eléctrica}

Además de transmisión, ERNC y diésel, se analizó la variación en la demanda como otro factor, motivándonos por el hecho de que el costo marginal se podría determinar por la diferencia de la oferta potencial del sistema y la demanda. 
La inclusión de las variables asociadas a la demanda (en términos absolutos o relativos) en nuestro modelo VAR no ayuda a explicar "el resto de los factores" de manera significativa, probablemente debido a la poca variación de esas variables en el período considerado.

Como muestra la Figura 2C, tampoco se encontraron diferencias significativas entre los períodos 2011-2013 y 2014-2016, afirmando que la demanda no fue un determinante de la caída de los costos marginales.

\section{FIGURA 2C}

\section{DESCOMPOSICION DE LA VARIANZA DEL COSTO MARGINAL: IMPACTO DE LA DEMANDA ELECTRICA}

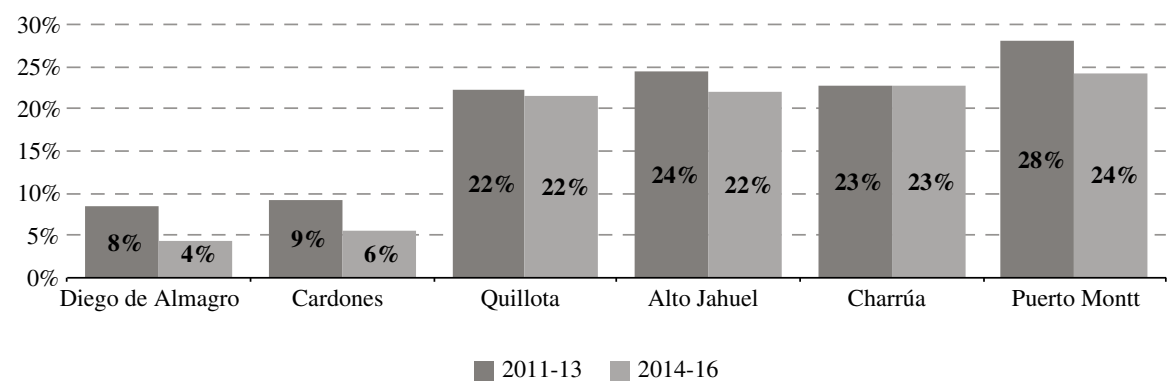

\section{D. Modelo con ERNC y diésel conjunto}

En el modelo C, el costo marginal depende de su propio rezago y de los aportes ERNC y aportes de diésel, mientras ERNC sigue un proceso independiente y aportes de diésel dependen de su propio rezago y de los aportes ERNC.

Este modelo combina los impactos de aportes ERNC y de aportes diésel (modelos A y B) y permite comparar las importancias relativas de los aportes ERNC y de diésel. Los resultados están presentados en Figura 2D.

Los resultados de este modelo son consistentes con los dos modelos anteriores. Para cada una de las seis barras consideradas, la importancia relativa de los aportes ERNC aumenta en los tres últimos años, mientras los aportes de diésel pierden su importancia. Debido a que hay más capacidad instalada ERNC con despacho prioritario, ya no hay tanta necesidad en despachar las centrales a diésel, y observamos que las tendencias en ERNC reemplazan el rol determinante que diésel tenía anteriormente, antes del 2014. 


\section{FIGURA 2D}

\section{DESCOMPOSICION DE LA VARIANZA DEL COSTO MARGINAL:} IMPACTOS ERNC Y DIESEL, MODELO C

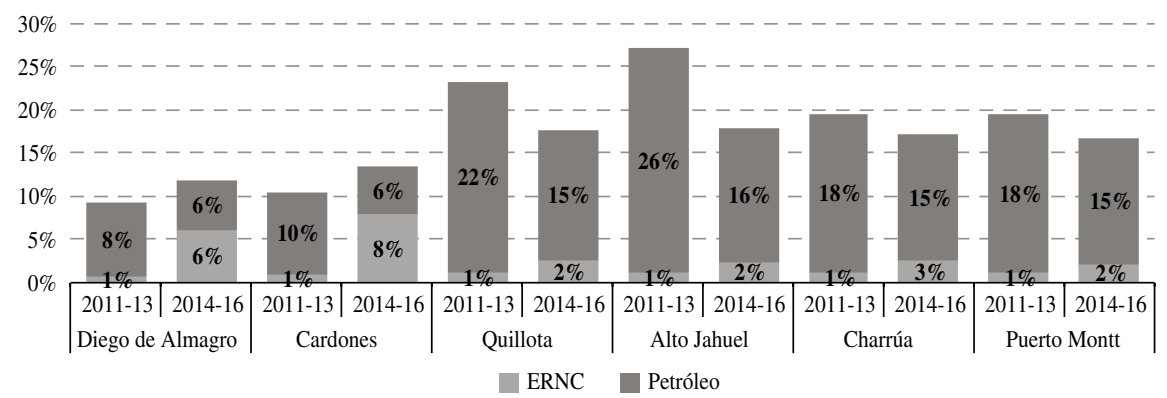




\section{ANEXO 3A \\ MODELO DE CORRECCION DE ERROR SIMPLE}

Otra manera de analizar la disminución del costo marginal es del punto de vista de relaciones de largo plazo entre el costo marginal y sus determinantes.

El modelo econométrico es el siguiente:

$$
\Delta x_{t}=\beta_{0}+\beta_{1} \Delta x_{t-1}+\beta_{2} \Delta x_{t-2}+\beta_{3} \Delta x_{t-3}+\gamma\left(x_{t}-y_{t}\right)+e_{t}
$$

Donde $\boldsymbol{x}$ corresponde al costo marginal, $\boldsymbol{y}$ corresponde al determinante del mismo. El error aquí se compone de dos partes: el error habitual $\boldsymbol{e}$ y la parte de la relación de largo plazo entre la variable de interés y su determinante.

La Tabla 3A abajo muestra los resultados de las regresiones de los 6 modelos de corrección del error simple. Se optó por tres rezagos para mantener consistencia entre el modelo VAR y los modelos de corrección de errores.

TABLA 3A

REGRESIONES CON TRES REZAGOS, CORRECCION DE ERROR SIMPLE

\begin{tabular}{|c|c|c|c|c|c|c|c|}
\hline Barra & CMg_L1 & CMg_L2 & CMg_L3 & Gas & Carbón & Hidro & Embalse \\
\hline DA & $\begin{array}{l}-0,076 \\
-0,078 \\
-0,085 \\
-0,093\end{array}$ & $\begin{array}{l}-0,212 * * * \\
-0,212 * * * \\
-0,218 * * * \\
-0,225 * * *\end{array}$ & $\begin{array}{l}-0,109^{*} \\
-0,112^{* *} \\
-0,117^{* *} \\
-0,122^{* *}\end{array}$ & $-0,034 *$ & $-0,022$ & $-0,011$ & $-0,002$ \\
\hline $\mathrm{CA}$ & $\begin{array}{l}-0,085 \\
-0,086 \\
-0,092 \\
-0,101 *\end{array}$ & $\begin{array}{l}-0,204 * * * \\
-0,204 * * * \\
-0,209 * * * \\
-0,216 * * *\end{array}$ & $\begin{array}{l}-0,124 * * \\
-0,126^{* *} \\
-0,131^{* *} \\
-0,137^{* *}\end{array}$ & $-0,033 *$ & $-0,022$ & $-0,012$ & $-0,002$ \\
\hline QU & $\begin{array}{l}0,082 \\
0,079 \\
0,080 \\
0,064\end{array}$ & $\begin{array}{l}-0,238 * * * \\
-0,241 * * * \\
-0,238 * * * \\
-0,251 * * *\end{array}$ & $\begin{array}{l}-0,100^{*} \\
-0,098^{*} \\
-0,098^{*} \\
-0,109^{*}\end{array}$ & $-0,048^{* *}$ & $-0,020$ & $-0,015$ & $-0,003^{*}$ \\
\hline $\mathrm{AJ}$ & $\begin{array}{l}0,105^{*} \\
0,102^{*} \\
0,103^{*} \\
0,085\end{array}$ & $\begin{array}{l}-0,263 * * * \\
-0,265 * * * \\
-0,263 * * * \\
-0,277 * * *\end{array}$ & $\begin{array}{l}-0,076 \\
-0,074 \\
-0,074 \\
-0,087\end{array}$ & $-0,053^{* * *}$ & $-0,023$ & $-0,017^{*}$ & $-0,003^{*}$ \\
\hline $\mathrm{CH}$ & $\begin{array}{l}0,111 * * \\
0,101 * \\
0,104 * \\
0,080\end{array}$ & $\begin{array}{l}-0,269 * * * \\
-0,279 * * * \\
-0,274 * * * \\
-0,292 * * *\end{array}$ & $\begin{array}{l}-0,118 * * \\
-0,121 * * \\
-0,120 * * \\
-0,138 * *\end{array}$ & $-0,074 * * *$ & $-0,024$ & $-0,021 * *$ & $-0,004 * *$ \\
\hline $\mathrm{PM}$ & $\begin{array}{l}0,110^{*} \\
0,095^{*} \\
0,100^{*} \\
0,070\end{array}$ & $\begin{array}{l}-0,261 * * * \\
-0,273 * * * \\
-0,267 * * * \\
-0,289 * * *\end{array}$ & $\begin{array}{l}-0,091 \\
-0,099^{*} \\
-0,096^{*} \\
-0,119^{*} *\end{array}$ & $-0,077 * * *$ & $-0,022$ & $-0,024 * *$ & $-0,005^{* * *}$ \\
\hline
\end{tabular}

